\title{
A multi-parent recombinant inbred line population of $C$. elegans allows identification of novel QTLs for complex life history traits
}

\author{
Basten L. Snoek ${ }^{1,2^{*}}$ D, Rita J. M. Volkers ${ }^{1}$, Harm Nijveen ${ }^{3}$, Carola Petersen ${ }^{6}$, Philipp Dirksen ${ }^{6}$, Mark G. Sterken ${ }^{1}$, \\ Rania Nakad ${ }^{6}$, Joost A. G. Riksen ${ }^{1}$, Philip Rosenstiel', Jana J. Stastna ${ }^{4}$, Bart P. Braeckman ${ }^{5}$, Simon C. Harvey ${ }^{4}$, \\ Hinrich Schulenburg ${ }^{6,8^{*}}$ and Jan E. Kammenga ${ }^{1^{*}}$
}

\begin{abstract}
Background: The nematode Caenorhabditis elegans has been extensively used to explore the relationships between complex traits, genotypes, and environments. Complex traits can vary across different genotypes of a species, and the genetic regulators of trait variation can be mapped on the genome using quantitative trait locus (QTL) analysis of recombinant inbred lines (RILS) derived from genetically and phenotypically divergent parents. Most RILs have been derived from crossing two parents from globally distant locations. However, the genetic diversity between local $C$. elegans populations can be as diverse as between global populations and could thus provide means of identifying genetic variation associated with complex traits relevant on a broader scale.

Results: To investigate the effect of local genetic variation on heritable traits, we developed a new RIL population derived from 4 parental wild isolates collected from 2 closely located sites in France: Orsay and Santeuil. We crossed these 4 genetically diverse parental isolates to generate a population of 200 multi-parental RILs and used RNA-seq to obtain sequence polymorphisms identifying almost 9000 SNPs variable between the 4 genotypes with an average spacing of $11 \mathrm{~kb}$, doubling the mapping resolution relative to currently available RIL panels for many loci. The SNPS were used to construct a genetic map to facilitate QTL analysis. We measured life history traits such as lifespan, stress resistance, developmental speed, and population growth in different environments, and found substantial variation for most traits. We detected multiple QTLs for most traits, including novel QTLs not found in previous QTL analysis, including those for lifespan and pathogen responses. This shows that recombining genetic variation across C. elegans populations that are in geographical close proximity provides ample variation for QTL mapping.
\end{abstract}

Conclusion: Taken together, we show that using more parents than the classical two parental genotypes to construct a RIL population facilitates the detection of QTLs and that the use of wild isolates facilitates the detection of QTLs. The use of multi-parent RIL populations can further enhance our understanding of local adaptation and life history trade-offs.

Keywords: Multi-parent RILs, C. elegans, QTL, Life-history, Natural variation, Genetic map

\footnotetext{
*Correspondence: I.b.snoek@uu.nl; hschulenburg@zoologie.uni-kiel.de; jan.kammenga@wur.nl

${ }^{1}$ Laboratory of Nematology, Wageningen University, Droevendaalsesteeg 1, NL-6708 PB Wageningen, The Netherlands

${ }^{6}$ Zoological Institute, University of Kiel, 24098 Kiel, Germany

Full list of author information is available at the end of the article
}

(c) The Author(s). 2019 Open Access This article is distributed under the terms of the Creative Commons Attribution 4.0 International License (http://creativecommons.org/licenses/by/4.0/), which permits unrestricted use, distribution, and reproduction in any medium, provided you give appropriate credit to the original author(s) and the source, provide a link to the Creative Commons license, and indicate if changes were made. The Creative Commons Public Domain Dedication waiver (http://creativecommons.org/publicdomain/zero/1.0/) applies to the data made available in this article, unless otherwise stated. 


\section{Background}

Determining genotype-phenotype relationships is the heart of genetics. Understanding how the relationships between traits, genotypes, and environments are controlled is also crucial to find traits relevant to the evolved context of the species [1-7]. The identification and characterization of allelic variants associated with complex traits have been a major challenge in plant and animal breeding as well as disease genetics. Many complex traits vary in a continuous way across different genotypes of a species. It is this phenotypic variation that can be mapped to the genome using quantitative trait locus (QTL) analysis. Standard QTL mapping for many different species is based on recombinant inbred lines (RILs) derived from a cross between two genetically and phenotypically divergent parents. One of the many species that has extensively been used for exploring the genetics of complex traits is the free-living, bacterivorous nematode Caenorhabditis elegans, reviewed in [8, 9].

C. elegans is found in most temperate regions in the world [10-12]. In the wild, this nematode is mostly found on rotten (plant) material and is also found associated with snails, slugs, and other invertebrates $[11,12]$. Populations of this opportunistic species can proliferate very fast (boom-and-burst). Generally, only a single $C$. elegans nematode reaches a new food source (e.g., rotting hogweed stem) and colonizes this resource. When food gets scarce, population growth halts and juveniles enter the long-lived dauer stage. In this stage, C. elegans nematodes display nictation behavior, allowing association with an invertebrate host [11, 13, 14]. Only a few juveniles of the colony will reach the next food source. Mostly, only a single genotype is found to dominate a food source, yet in some cases, multiple genotypes are found to co-occur [15]. Generally, a single genotype is dominant in an area but can be replaced over time $[15,16]$.

In nature, $C$. elegans reproduces primarily through self-fertilization although outcrossing with males occurs occasionally [15-18]. Individual worms typically produce up to 350 offspring when self-fertilizing, and the generation time of $C$. elegans is 3.5 days (at $20^{\circ} \mathrm{C}$ ). Outcrossing could be hampered due to genetic incompatibilities $[19,20]$ and possibly outbreeding depression [21]. Both effects could account for the low frequency of heterozygous $C$. elegans nematodes in nature and the dominance of a single genotype in most areas [15-18]. At the same time, a certain level of genetic diversity, at least at single loci, may be maintained by balancing selection, which has been repeatedly identified in natural $C$. elegans populations, for example, in relation to genetic mating incompatibility [19], virus resistance [22], and foraging behavior [23]. Both outbreeding depression and balancing selection may favor epistatic interactions among loci, which jointly influence the traits under selection, further shaping the genetic composition of natural worm populations. Moreover, $C$. elegans population genetics are also shaped by the boom-and-bust lifestyle of this nematode species, which is particularly common in ephemeral habitats, where it is subject to frequent extinction and re-colonization events (reviewed in [11, 12]). As a consequence of these different dynamics and selective forces, genetic diversity between $C$. elegans populations on a local scale can be almost as diverse as on a global scale, with genetically distinct populations occurring within a few kilometers distance $[10,17$, $18,24,25]$. The analysis of RILs, which are constructed from natural isolates, may then help us understand at least some of the involved dynamics, especially as to the traits under selection and the underlying genetic architecture. Furthermore, the generation of RILs will break up strong interacting loci, possibly revealing the underlying genetic architecture of complex traits [26, 27], as exemplified by the dissection of genetic incompatibility loci $[19,28]$. Creating a population of RILs from a cross between multiple locally isolated C. elegans haplotypes and thus generation of multi-allelic genetic mosaics enables the exploration of the link between lifecycle dynamics, genetic variation, and evolutionary processes.

Most inbred mapping populations of $C$. elegans were derived from two globally distant locations, namely Bristol, UK (N2 strain) and Hawaii (CB4856 strain) [9, 29-32]. These Bristol-Hawaii RIL populations have been very valuable for studying the genetic architecture of complex traits [33-45] and the identification of the underlying genes [23, 46-56], even though other genotypes have been used for the construction of RIL populations, e.g., crosses between N2 and BO [57], N2 and DR1350 [43], N2 and LSJ1 [58], JU605 and JU606 [59], MT2124 and CB4856 [60], and JU1395 and MY10 [61]. Typically, these additional RILs have only been used to address a specific research question, and thus, their suitability to map QTLs for different types of traits is unclear compared to the work on the Bristol-Hawaii populations. Although other types of crossing strategies involving multiple lines [62] or panels of wild isolates have been reported, most of the work has been done on the Bristol-Hawaii-derived RILs which only captures a subset of the phenotypic and genetic diversity present in C. elegans.

The inclusion of more than 2 parental sources of genetic variation and alleles captures more genetic variation and allows for more precise mapping and identification of potential regulatory variants of complex traits [63]. An alternative to the conventional 2 parental genetic mapping strategies is the development of Multiparent Advanced Generation Inter-Cross (MAGIC) lines. The first of such populations was developed for Arabidopsis thaliana consisting of 527 RILs developed from 19 different parental accessions [64]. Since then, many more MAGIC populations have been developed for a range of 
species [65]. Recently, a C. elegans multi-parental RIL population originating from 16 wild types that underwent almost 200 generations of experimental evolution [62] was characterized [26]. This RIL panel comprised of 507 strains covers about $22 \%$ of single nucleotide polymorphisms (SNPs) known to segregate in natural populations $[26,62]$.

Here, we report the construction and analysis of a multi-parental (4) recombinant inbred line (mpRIL) population for C. elegans. The $200 \mathrm{mpRILs}$ are derived from an advanced cross between 4 wild types: JU1511 and JU1941 isolated from Orsay (France) and JU1926 and JU1931 isolated from Santeuil (France) (kindly provided by MA Félix, Paris, France; [25]). The RILs were SNP genotyped based on RNA-seq data. We used the SNP-genotyped lines for mapping QTLs for the following C. elegans phenotypes: length, width, length to width ratio, volume, lifespan, lifespan during dietary restriction, heat-shock survival, oxidative stress, occurrence of males, the developmental speed on the food sources of Escherichia coli OP50 and naturally $C$. elegans-associated bacteria Erwinia rhapontici $[25,66]$, and population growth on $E$. coli OP50 and naturally C. elegans-associated bacteria Erwinia rhapontici, Sphingomonas sp., non-pathogenic Bacillus thuringiensis strain DSM-350E, and the pathogenic Bacillus thuringiensis strain NRRL B-18247 [25]. We aimed to measure a range of traits under different bacterial food conditions and abiotic conditions that, to a certain extent, reflect natural conditions [12, 25, 67]. For all these traits, heritable variation and QTLs were found. Here, we present a new multi-parental recombinant inbred line population and show the distribution of genetic variation, recombination, trait variation, and identified quantitative trait loci and show the effects of local genetic variation on phenotypic traits.

\section{Results}

Developing a C. elegans multi-parental recombinant inbred line population

To allow the 4 parental genomes (JU1511, JU1941, JU1926, and JU1931) [25] to recombine, we set up a crossing scheme in which 2 pairs of wild isolates were crossed and both the obtained F1 populations were reciprocally inter-crossed (Fig. 1; Additional file 1: Table S1). To enable crossovers on chromosome $\mathrm{X}$ and to generate extra crossovers, the heterozygous F2 obtained from these initial crosses were further inter-crossed. To create homozygous genotypes, single worms were selected from the F2 as well as from the F2 inter-cross for 6 generations of single-worm inbreeding. From these 383 lines, a population of 200 different multi-parental recombinant inbred lines (mpRILs) was

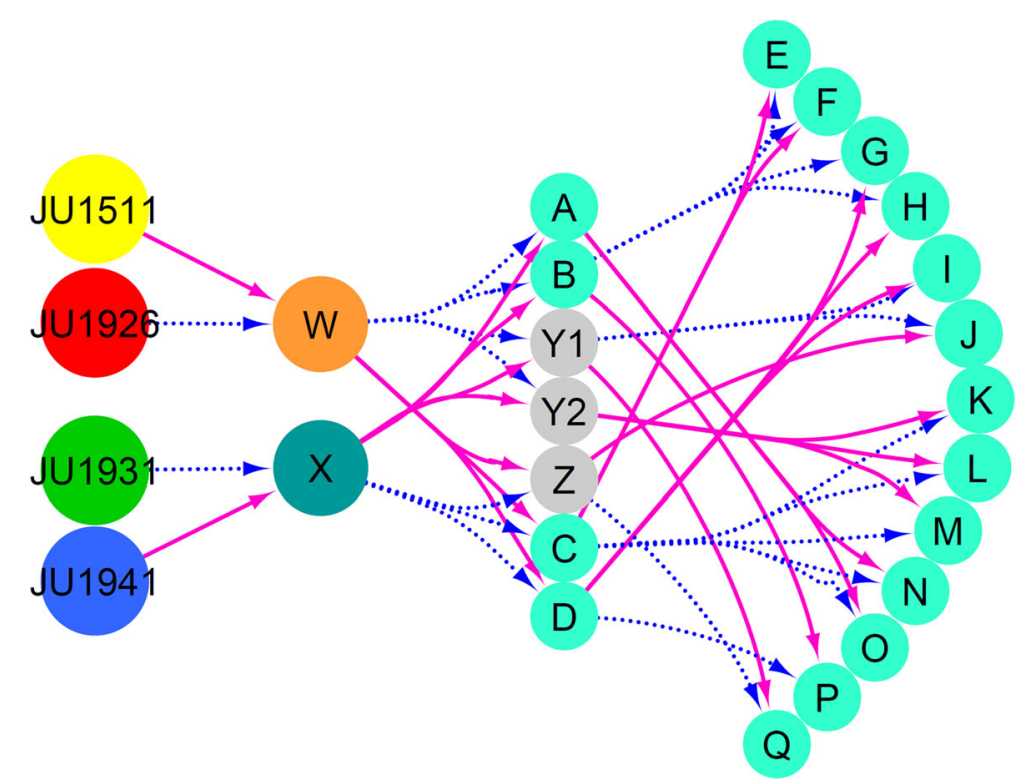

Fig. 1 Crossing scheme used to make the four parental mpRIL population. Different populations of worms are shown in the colored circles. Magenta solid lines indicate the hermaphrodite parental lines, and the dashed blue lines show the male parental lines. A JU1511 hermaphrodite (yellow) was crossed with a JU1926 male (red) to create F1 population W. A JU1941 hermaphrodite (blue) was crossed with a JU1931 male (green) to create F1 population X. Individuals from populations $W$ and $X$ were reciprocally crossed to create seven separate populations. In this way, we obtained different populations of genotypes with mixed genetic background from the four parental lines. Individuals from the seven separate populations ( $A, B, Y 1, Y 2, Z, C$, and D) where further intercrossed to obtain extra recombinations mainly to break up the X-chromosome, which lacks recombination in the male. These populations are labeled $\mathrm{E}$ to $\mathrm{Q}$. From these populations (light blue), individuals were taken for inbreeding via self-fertilization for six generations to create mpRILs. For details, see also Additional file 1: Table S1 and Fig. 3 
randomly picked for mRNA sequencing to obtain the genetic variation in the coding sequence.

\section{Polymorphisms are not distributed equally}

For genotyping of the $200 \mathrm{mpRILs}$, we used the single nucleotide polymorphisms (SNPs) obtained from RNA-seq. We detected 8964 SNPs diverging in the coding sequences between the parental lines. The distribution of these SNPs over the genotypes can be grouped into 7 specific $S N P$ distribution patterns (SDP): 4 patterns represent the 4 parental strains; 3 patterns are shared between 2 parental strains versus the other 2 parental strains. These are SDP 12-difference between pair JU1511/JU1926 and pair JU1931/JU1941; SDP 13-difference between pair JU1511/ JU1931 and pair JU1926/JU1941; and SDP 14-difference between pair JU1511/JU1941 and pair JU1926/JU1931. Importantly, SDP 14 therefore represents SNPs diverging between the 2 isolation sites, and hence, these polymorphisms may be informative of local adaptation. The SNP distribution differed between parents, and the SNPs were unequally distributed across the genome (Table 1; Fig. 2; Additional file 1: Table S2). For example, chromosomes I, III, and $\mathrm{V}$ were more polymorphic in their coding sequences compared to chromosomes II, IV, and X. Overall, chromosome II was the least polymorphic and chromosome III was the most polymorphic. Furthermore, we found regions where multiple SDP overlap (left arm of chromosomes I, IV, and V; right arm of chromosomes I, IV, V, and X, and all of chromosome III; Fig. 2), which can potentially be used to reduce the number of candidate causal SNPs.

Most SNPs were strain specific or diverging between strains from the two isolation sites. For example, unique SNPs on chromosome I were mostly specific for JU1941 alleles, while those on chromosome V were mostly specific for JU1931. For JU1511, unique SNPs were found

Table 1 SNP distribution patterns (SDP). Distribution of SNPS and alleles in total and per chromosomes I to X for each SDP. Strain names and place of isolation are give in the column headers. At SDP 14, O vs S shows those SNPs different between the two strains isolated from Orsay and the two strains isolated from Santeuil

\begin{tabular}{lllllllll}
\hline & Total & $\begin{array}{c}\text { JU1511, } \\
\text { Orsay }\end{array}$ & $\begin{array}{l}\text { JU1926, } \\
\text { Santeuil }\end{array}$ & $\begin{array}{l}\text { JU1931, } \\
\text { Santeuil }\end{array}$ & $\begin{array}{l}\text { JU1941, } \\
\text { Orsay }\end{array}$ & $\begin{array}{l}\text { SDP } \\
12\end{array}$ & $\begin{array}{l}\text { SDP } \\
13\end{array}$ & $\begin{array}{l}\text { SDP } \\
14, \text { O } \\
\text { vs S }\end{array}$ \\
\hline Total & 8964 & 2628 & 748 & 1651 & 2137 & 313 & 842 & 518 \\
I & 1916 & 288 & 10 & 75 & 1250 & 131 & 109 & 41 \\
II & 503 & 254 & 229 & 4 & 4 & 0 & 2 & 3 \\
III & 2231 & 979 & 16 & 217 & 476 & 72 & 281 & 158 \\
IV & 930 & 178 & 447 & 91 & 14 & 1 & 12 & 162 \\
V & 2152 & 104 & 11 & 1257 & 214 & 88 & 303 & 144 \\
X & 1232 & 825 & 35 & 7 & 179 & 21 & 135 & 10 \\
\hline
\end{tabular}

on all chromosomes whereas the other parental genotypes have chromosomes almost completely lacking unique SNPs. Moreover, the genotypes from Orsay (JU1511 and JU1941) had more (> 2000) strain-specific SNPs compared to those from Santeuil (JU1926 and JU1931). Almost 1700 SNPs were found in Orsay vs. Santeuil genotypes, whereas only $518(\sim 30 \%)$ were shared between genotypes from the same geographical location.

Across all chromosomes, both the left and right arms of the chromosomes (except for chromosome III) were more polymorphic compared to the center of the chromosomes, a result matching that seen in previous work on C. elegans wild isolates $[10,29,68]$. Specific regions were very polymorphic between the four parental lines, such as the left arm of chromosome I, all of chromosome III, both arms of chromosome V, and the right arm of chromosome X. Long stretches of relative low SNP variation could also be observed, such as large parts of chromosome II, middle part of chromosome IV, and left arm of chromosome X. For the majority of the genome, at least one parental genotype could be uniquely identified by individual SNPs. Overall, we concluded that SNPs in coding regions of C. elegans were unequally distributed over the genome and among genotypes and that the chromosome arms were more polymorphic than the chromosome centers.

\section{Cross-specific recombination in the mpRILs}

The genetic map shows a highly variable frequency of recombination and introgression sizes (Fig. 3a; Table 2; Additional file 1: Tables S3 and S4). In total, 1683 recombination events were found in the mpRIL population, with a genome-wide allelic presence of the 4 parental lines (Fig. 3b, Additional file 2: Figure S1). Up to 4 recombination events per mpRIL per chromosome were found, where 1 or 2 recombination events per chromosome was most common (Fig. 3c). The average number of crossovers per mpRIL was 8.5 across all chromosomes and 1.5 per chromosome. Most recombination events were found on chromosome III (348). As expected, due to the lack of recombination of chromosome $\mathrm{X}$ in males, the fewest recombination events were found on chromosome X (233) (Fig. 3d). Moreover, for chromosome $\mathrm{X}$, almost $40 \%$ of the mpRILs showed no recombination. The recombination rate was on average 1 per $17 \mathrm{Mb}$, with a genome-wide mean introgression size of 5.0 Mbp (median 3.1 Mbp). We observed a suppression of recombination across the centers of the chromosome with higher recombination rates at the chromosome arms (Fig. 3e), consistent with previous work on C. elegans [10, 29, 69]. Considering the whole population, the genomic bins (loci) that could be individually investigated had a median size of $43 \mathrm{kbp}$. (Table 2). The effective recombination rate useful for QTL mapping becomes larger as 


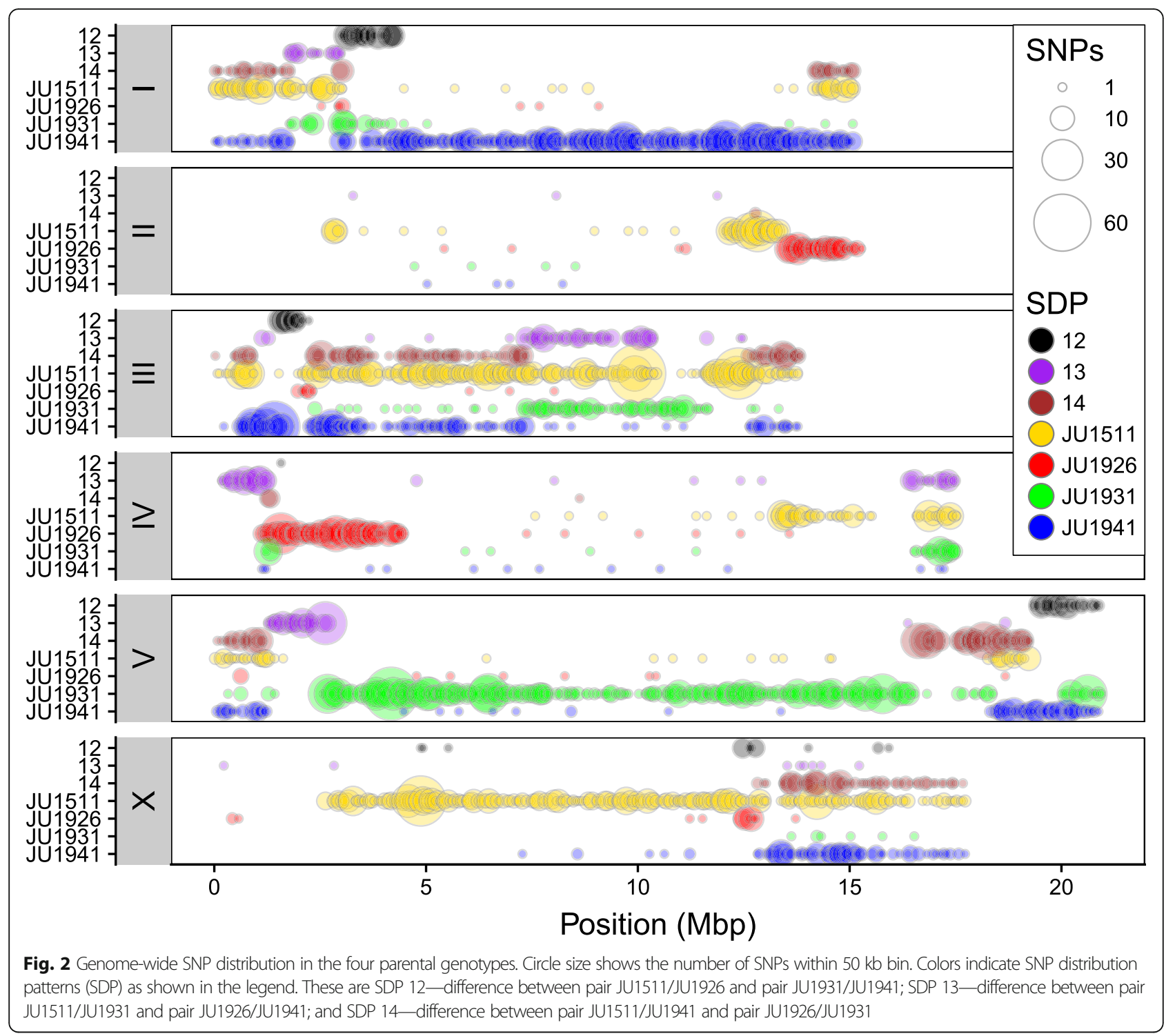

multiple SDP could be recombined by a single recombination event (Additional file 3: Figure S2). Including the SDP increased the effective recombination rate to approximately 57 per Mbp and 5686 recombination events in total. This did not affect the mapping resolution (making the QTLs smaller), yet it did reduce the amount of potentially causal polymorphisms, and therefore, mapping in an SDP-dependent manner affected the number of polymorphisms under investigation when looking for the causal gene or SNP.

The allelic distribution was different between cross and inbreeding pools. The ratio of parental alleles shows a similar distribution across the chromosomes, except for chromosome II (Fig. 3b). Alleles from all four parents had a genome-wide representation, although JU1931 alleles occurred less frequently genome-wide and JU1926 alleles occurred relatively less frequently on chromosomes I, III, and X. The allelic distribution was dependent on the specific cross and inbreeding pool (Fig. 1 and Additional file 2: Figure S1; Additional file 1: Table S1). In each specific pool, the parental alleles display a cross-specific and chromosome-specific distribution, frequently showing an absence of one or a few allele types (Additional file 2: Figure S1). Taken together, the whole population of mpRILs captures the genetic variation of the parental strains from which they were derived, perturbed by recombination.

\section{Phenotypic variation and heritability}

The studied phenotypes were chosen based on previous work on these $C$. elegans strains where we describe extensive phenotypic variation between the parental lines [25]. Moreover, we focused on a variety of traits that are generally relevant for the ecology and evolutionary fitness of $C$. 

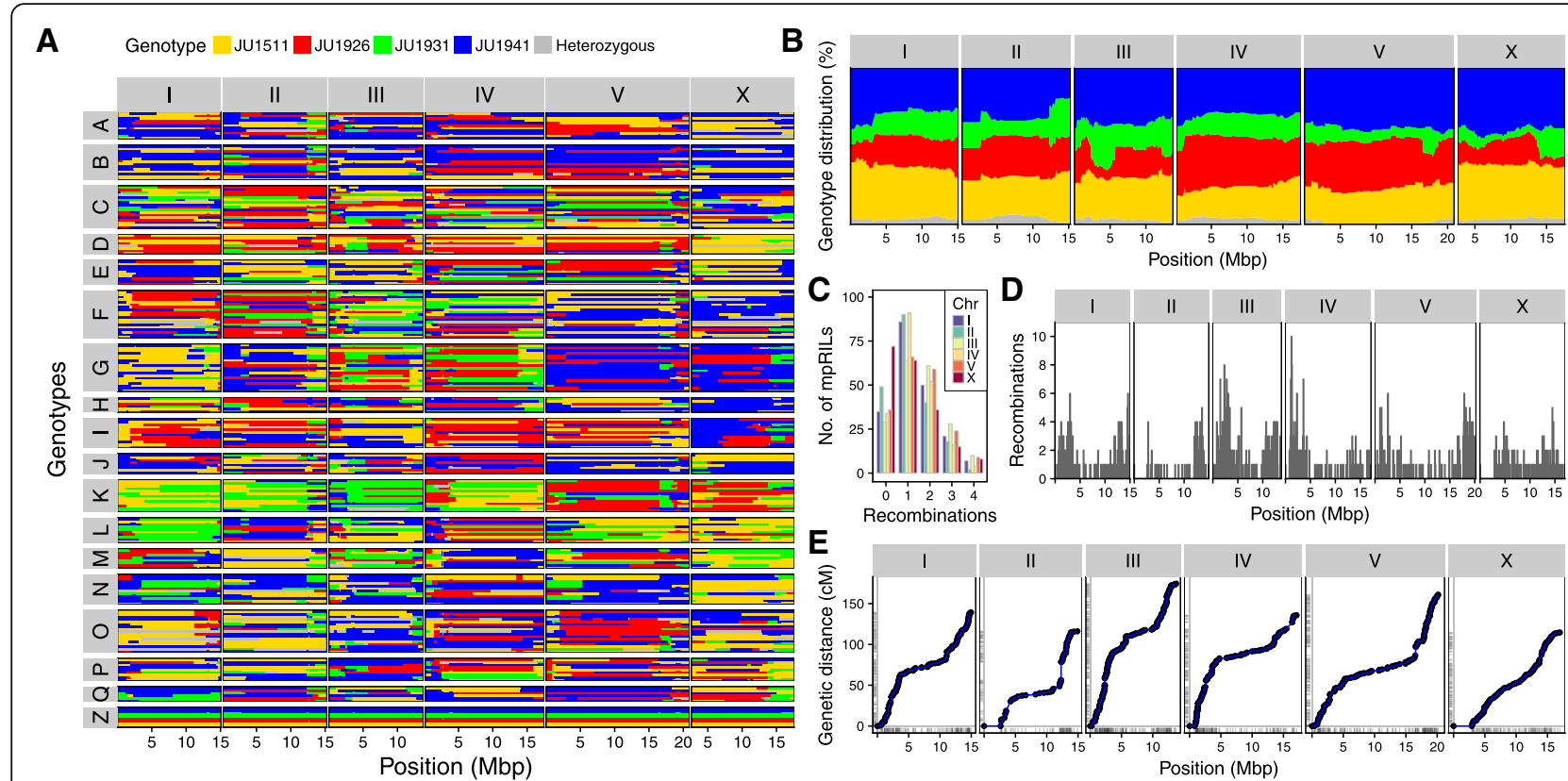

Fig. 3 Parental background of the multi parental recombinant inbred lines and recombination and allelic distribution per chromosome. a Colors indicate the parental background per genetic segment ( $x$-axis) per RIL ( $y$-axis) as estimated from the SNP distribution patterns. Chromosomes are in separate panels on the $x$-axis. mpRILs are grouped according to their cross history. The parental lines are shown in group Z. b Genome-wide distribution of parental alleles. Colors indicate the percentage of parental occurrence ( $y$-axis) per genetic segment ( $x$-axis) as estimated from the SNP distribution patterns. Chromosomes are in separate panels on the $x$-axis. c Recombination per chromosome. Chromosomes show on the $x$-axis. Numbers of recombinations per RIL are shown on the $y$-axis. $\mathbf{d}$ Recombination frequency per chromosome. e Genomic distance $(x)$ vs genetic distance $(y)$, the rug lines (small lines on the axis) indicate the marker positions

elegans in nature. Even though the range of traits is non-exhaustive, they cover distinct ecologically important characteristics, including population growth, lifespan, speed of development, and stress resistance. We specifically considered some traits that are likely under high selection in nature, such as the response to natural food microbes, in case of Erwinia and Sphingomonas, isolated together with the parental strains [25] or pathogens, which we assessed using population growth as a meaningful proxy for worm fitness. For most traits, we observed substantial heritable phenotypic variation between mpRILs (Fig. 4; Additional file 1: Table S5 and Table S6). Correlation analysis (Fig. 5; Additional file 1: Table S7) across all phenotypic traits showed that the timing of the first eggs laid was highly correlated across different food conditions. This was also found for population growth, except growth on Sphingomonas. Body size and developmental phenotypes were also highly correlated, showing that these phenotypes were likely to share a similar genetic architecture.

We measured 21 phenotypes in the mpRILs of which 17 also in the parental wild types. Average lifespan was 18 days (range 13 to 26 days) and $\sim 1$ day longer under dietary restriction (DR) at 19 days (range 13 to 27 days). The overall effect of DR on lifespan was positive, but negative effects were observed for individual genotypes (approximately -6 days to approximately +7 days) as previously found in C. elegans [70] and in mice [71]. Heat shock $\left(10 \mathrm{~h}\right.$ at $\left.35{ }^{\circ} \mathrm{C}\right)$ had a severe effect on the

Table 2 Crossovers, introgression size and bin size in total and per chromosome

\begin{tabular}{|c|c|c|c|c|c|c|c|}
\hline & Total & Average per mpRIL & No. of mpRILs without COs & No. of mpRILs with COs & $\begin{array}{l}\text { Mean introgression } \\
\text { size (Mbp) }\end{array}$ & $\begin{array}{l}\text { Median introgression } \\
\text { size (Mbp) }\end{array}$ & $\begin{array}{l}\text { Median bin } \\
\text { size (kbp) }\end{array}$ \\
\hline Total & 1683 & 8.5 & 1 & 198 & 5.0 & 3.1 & 43.1 \\
\hline । & 277 & 1.4 & 35 & 164 & 4.9 & 3.1 & 45.4 \\
\hline$\|$ & 232 & 1.2 & 49 & 150 & 4.4 & 3.6 & 44.3 \\
\hline III & 348 & 1.7 & 29 & 170 & 4.2 & 2.9 & 26.2 \\
\hline IV & 272 & 1.4 & 34 & 165 & 6.0 & 3.4 & 45.1 \\
\hline V & 321 & 1.6 & 36 & 163 & 6.3 & 3.7 & 43.1 \\
\hline$x$ & 233 & 1.7 & 72 & 127 & 3.8 & 2.5 & 68.9 \\
\hline
\end{tabular}



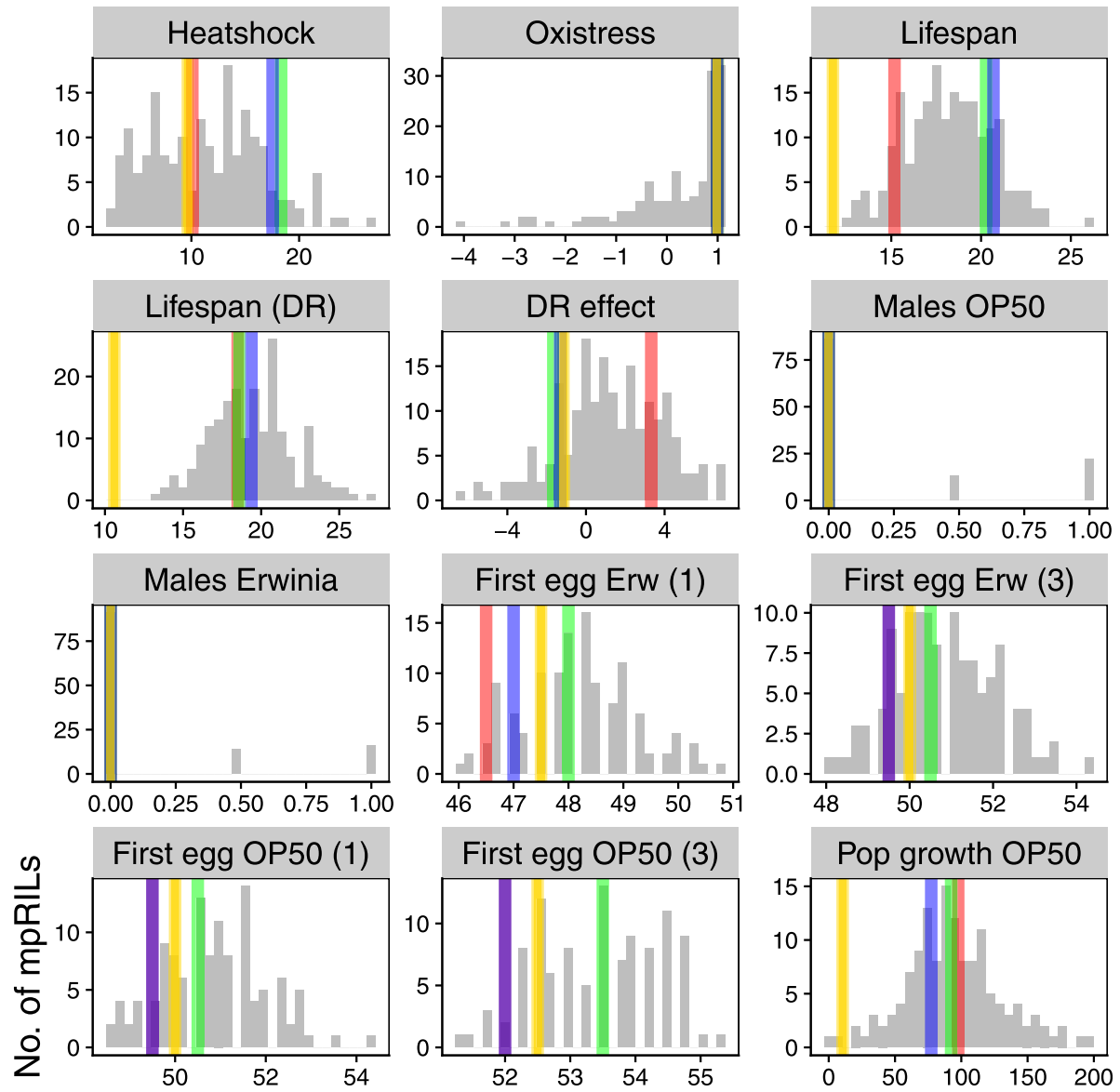

First egg OP50 (3)
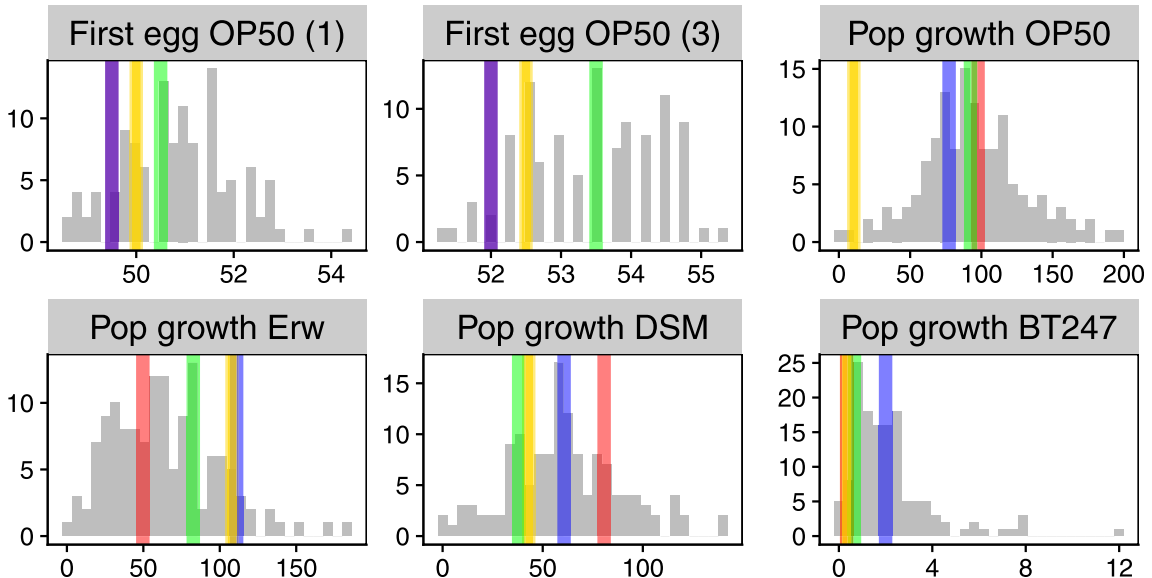

Pop growth DSM
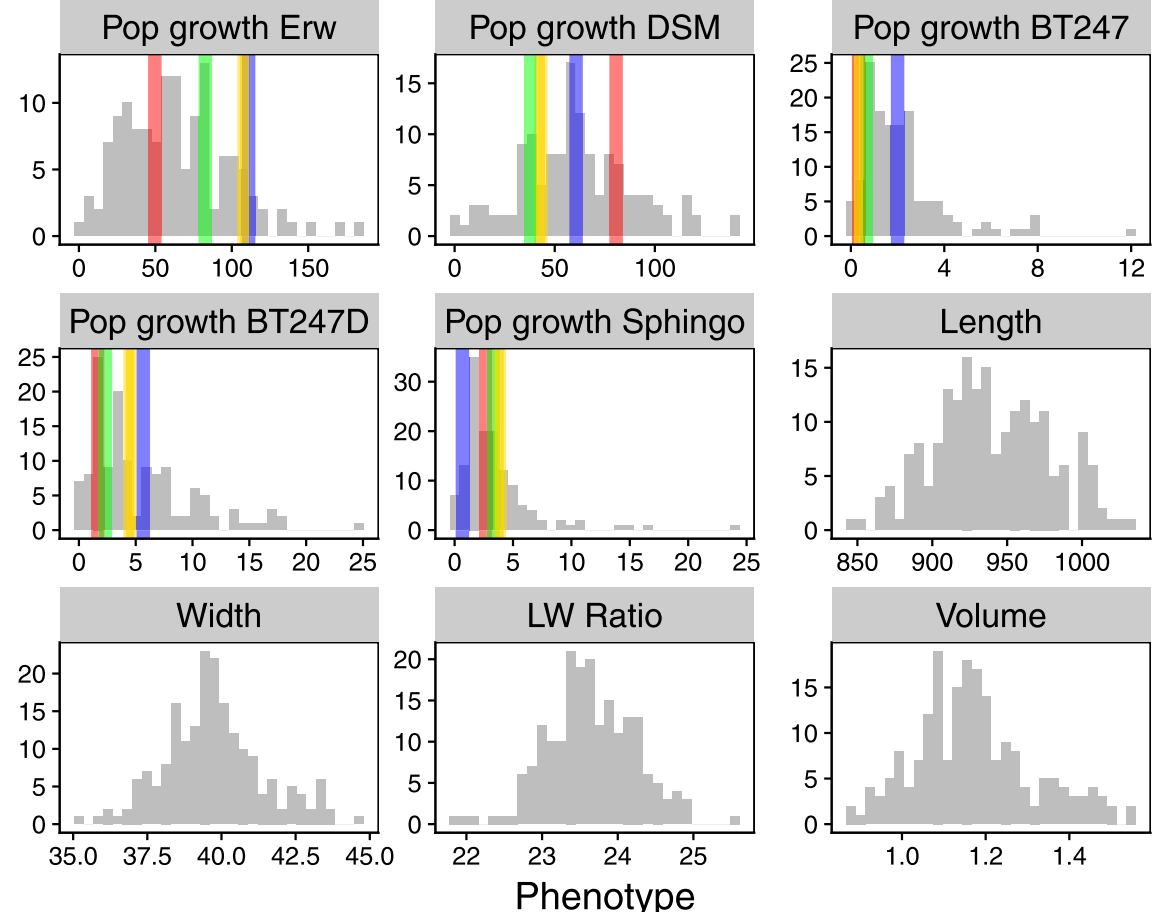

Fig. 4 (See legend on next page.) 
(See figure on previous page.)

Fig. 4 Phenotypic variation in the mpRILs. On the $y$-axis, the number of mpRILs is shown. The $x$-axis values depend on the trait. Heat shock is the average number of dead animals per 50. Oxidative stress indicates activity. Lifespan is the average lifespan on NGM in days. Lifespan (DR) is the average lifespan on DR medium in days. DR effect is the difference in the average lifespan between NGM and DR medium in days. Males OP50 and males Enwinia is the occurrence of males on plates $(0=$ none , $0.5=1$ plate, $1=2$ plates). First egg Erw (1) is the time in hours until the first egg (1-10) for populations grown on Erwinia. First egg Erw (3) is the time in hours until the first egg (> 100) for populations grown on Enwinia. First egg OP50 (1) is the time in hours until the first egg (1-10) for populations grown on OP50. First egg OP50 (3) is the time in hours until the first egg (> 100) for populations grown on OP50. Pop growth shows worms per $5 \mu$ of culture. Length and width in nanometers, LW ratio is the ratio between the length and width; volume in nanoliters. The parental values for these traits are not shown yet were measured in a different batch in Volkers et al. 2013. Distribution in the mpRILs are shown in gray, and parental strains are shown JU1511 (yellow), JU1926 (red), JU1931 (green), and JU1941 (blue)

survival, on average $\sim 11 \%$ (3 to 27 ) of the population survived 2 days after. Oxidative stress did not affect the average behavioral activity but did have an effect on individual genotypes ( -4 to -1$)$. Worms fed on Erwinia started laying their eggs earlier compared to worms fed on OP50. As previously found [25, 72], the average time of the first egg laid on Erwinia was shorter (mean $\sim 51 \mathrm{~h}$; range $\sim 48$ to $\sim 54 \mathrm{~h}$ ) than on OP50 (mean $\sim 53 \mathrm{~h}$; range $\sim 51$ to $\sim 55 \mathrm{~h}$ ). The occurrence of males was similar on both OP50 and Erwinia; for most mpRILs, no males were found, yet the genotypes that had males in the population did so on both OP50 and Erwinia. Population growth of the mpRILs differed strongly between worms fed with different bacteria as previously found between wild isolates [25]. On average, population growth was highest on OP50 (mean 93 individuals $/ 5 \mu \mathrm{l}$; range 0 to $197 / 5 \mu \mathrm{l}$ ), on Erwinia (mean $\sim 65 / 5 \mu \mathrm{l}$; range 0 to $183 / 5 \mu \mathrm{l}$ ), and DSM (mean $\sim 61 / 5 \mu \mathrm{l}$; range 0 to $140 / 5 \mu \mathrm{l}$ ). Slow growth was observed on Sphingomonas (mean $\sim 3 / 5 \mu \mathrm{l}$; range 0 to 24 / $5 \mu \mathrm{l}$ ) and on BT247 (mean $\sim 2 / 5 \mu \mathrm{l}$; range 0 to $12 / 5 \mu \mathrm{l}$ ). The mpRILs are also variable in length (mean $945 \mu \mathrm{m}$; range 848 to $1135 \mu \mathrm{m}$ ), width (mean $40 \mu \mathrm{m}$; range 35 to $46 \mu \mathrm{m}$ ), length to width ratio (mean 24; range 22 to 26 ), and volume (mean $1.2 \mathrm{nl}$; range 0.9 to $1.9 \mathrm{nl}$ ).

The highest heritability (83\%) was found for population growth on Erwinia, whereas the lowest (55\%) was found

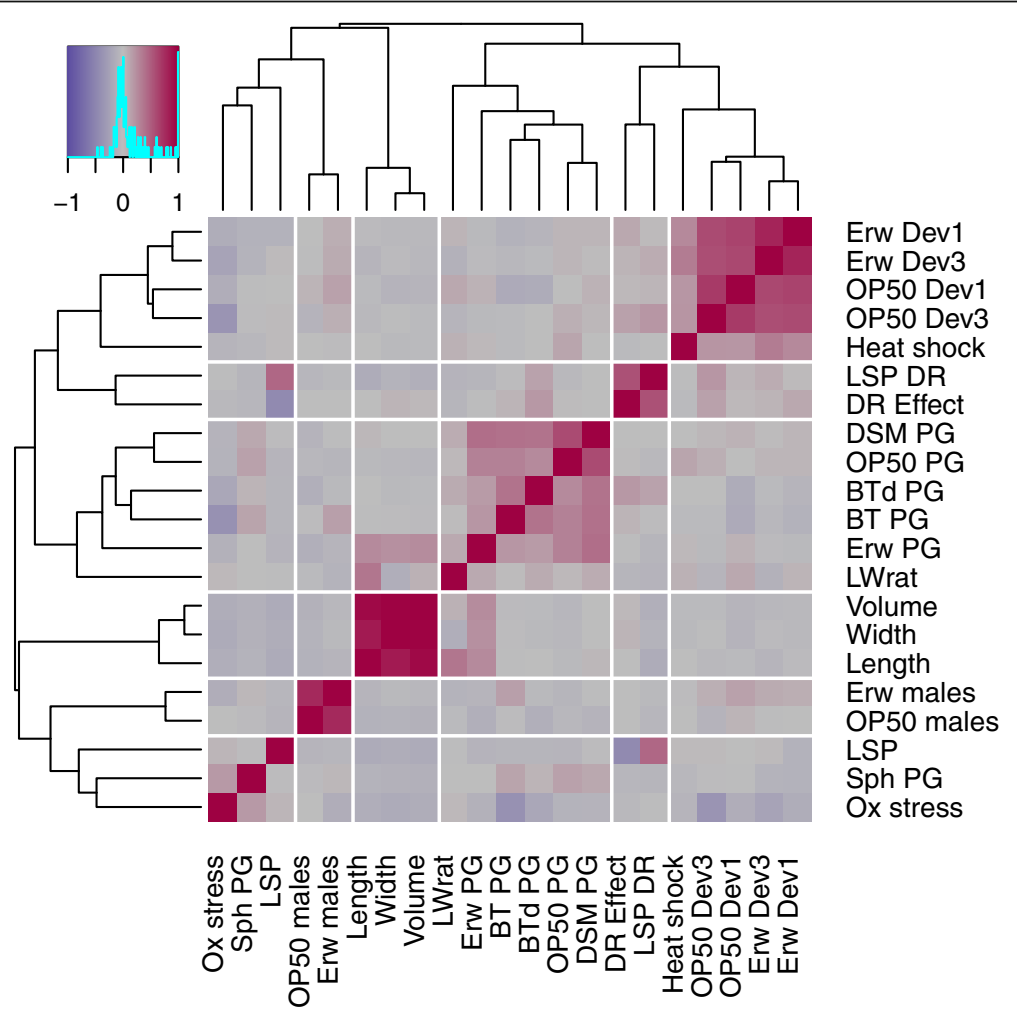

Fig. 5 Correlations between traits in the mpRILs. Ewr Dev1 and Ewr Dev3 are the speed of development on Erwinia as measured by time until the first egg $(1=$ first egg, 3 = first $>100$ eggs). OP50 Dev1 and OP50 Dev3 are the speed of development on OP50. Heat shock is the alive worms after $10 \mathrm{~h}$ of $35^{\circ} \mathrm{C}$. LSP DR is the lifespan on dietary restriction. DR Effect is the difference in lifespan between LSP DR and LSP. DSM PG, OP50 PG, BTd PG, BT PG, and Erw PG are the population growth on bacteria DSM, OP50, BT diluted, BT and Enwinia, respectively. LWrat is the length to width ratio. Volume is the animal volume. Width is the animal width. Length is the animal length. Erw males and OP50 males are the occurrence of males on Erwinia and OP50, respectively. LSP is the mean lifespan. Sph PG is the population growth on Sphingmonas. Ox stress is the oxidative stress survival 
on the most toxic concentration of BT247. Developmental speed for both OP50 and Erwinia showed high heritability (80\%) with many mpRILs having phenotypes beyond the parental phenotypic values (Additional file 1: Table S6). Most mpRILs developed slower than the parents. Body size also showed high heritability ( $80 \%)$, with a length to width ratio of $\sim 70 \%$. Population growth on the different bacteria showed variation in heritability, possibly linked to average growth rate on the specific bacteria. Transgression shows mpRILs beyond the parental phenotypes on both sides, yet for the growth on the BT247 strain, transgressive mpRILs mostly produce better growth than the parents.

Together, the results show that ample phenotypic variation of complex traits can be found between the mpRILs and that these phenotypic differences are heritable. Genetic variation across the mpRILS causal for these different functional differences is likely to have fitness effects.

\section{Quantitative trait loci}

By applying quantitative trait locus (QTL) mapping using a forward co-factor selection approach, we identified the loci associated with variation in the measured phenotypic traits (Figs. 6 and 7; Additional file 1: Table S8; Additional file 4: Figure S3). The average QTL interval was 1.2 Mbp, median QTL interval was $0.88 \mathrm{Mbp}$, minimum QTL interval was $2.06 \mathrm{kbp}$, and maximum QTL interval was 7.7 Mbp. Most QTLs were found for the lifespan/stress traits (3-7 per trait), and together, these explained between 32 up to $41 \%$ of the total trait variation observed. Of the 21 lifespan/stress QTLs, most showed an allelic difference between JU1941 (7) or JU1511 (6) and the other 3 parental genotypes. The most significant QTL was found on chromosome $\mathrm{X}$ at $\sim 16 \mathrm{Mbp}$ for the effect of oxidative stress which explained $20 \%$ of the variation. For developmental speed, 2 to 3 QTLs were found per trait and together explained 24 to $31 \%$ of the total variation per trait. Again, most of the 9 QTLs found for all developmental speed traits showed an allelic difference between JU1941 (3) or JU1511 (5) and the rest. Of all these QTLs, a QTL found on chromosome III around 12.3 Mbp (for which the JU1511 allele shortens the developmental speed on Erwinia by almost $1 \mathrm{~h}$ ) explained most variations

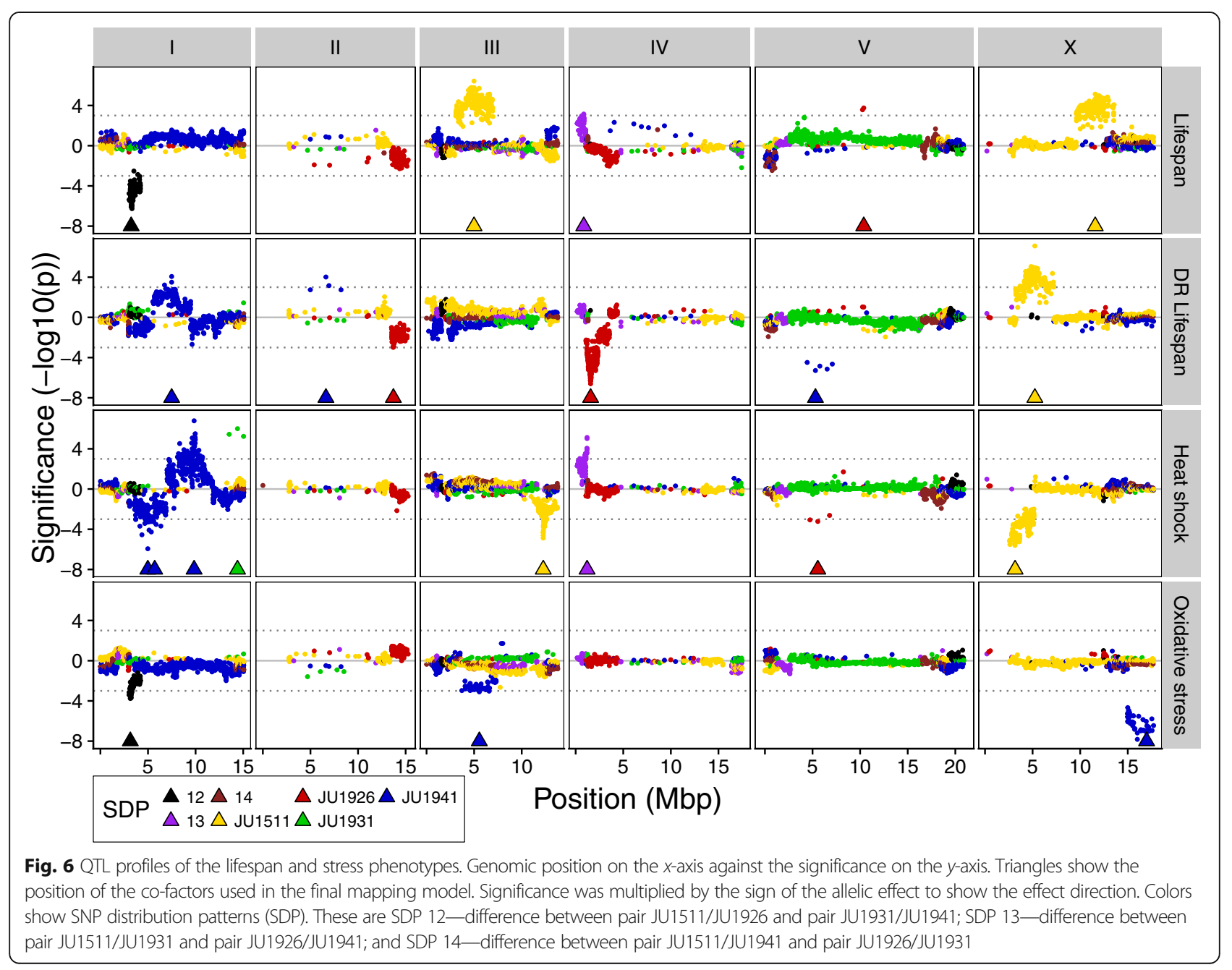




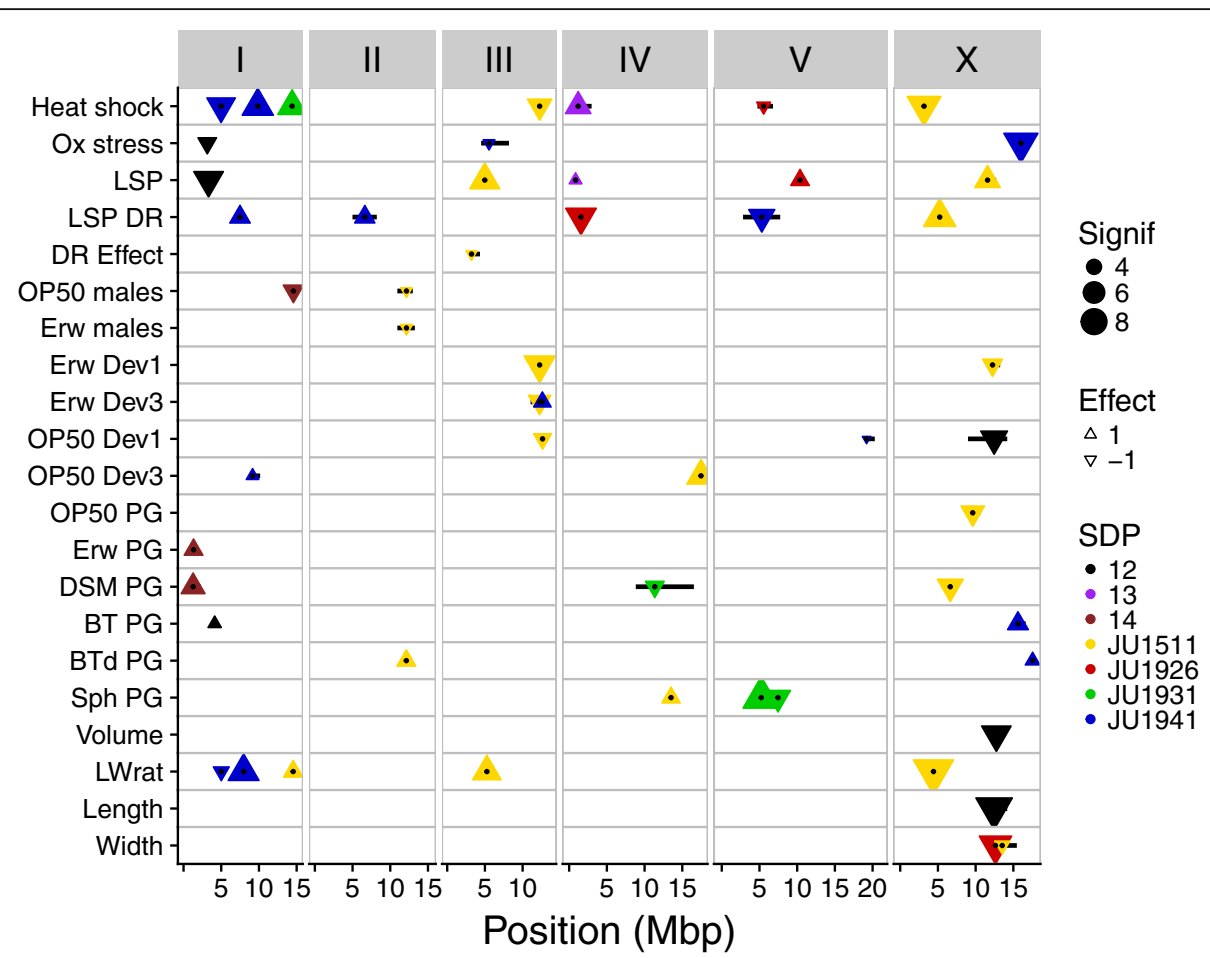

Fig. 7 Genome-wide overview of QTLS. QTLs are shown by the triangles; triangles pointing upwards show a positive allelic effect, and triangles pointing downwards show a negative allelic effect. Size indicates significance in -log10(p); colors show SNP distribution patterns (SDP). These are SDP 12-difference between pair JU1511/JU1926 and pair JU1931/JU1941; SDP 13-difference between pair JU1511/JU1931 and pair JU1926/ JU1941; and SDP 14-difference between pair JU1511/JU1941 and pair JU1926/JU1931. Black dots show the exact location of the peaks and the black horizontal bars the $2-\log 10(p)$ drop QTL intervals

(22\%). Population growth traits showed between 1 and 3 QTLs, where the 3 QTLs for both Sphingomonas and DSM explained $\sim 30 \%$ of the variation. The growth on other bacteria had less than $16 \%$ of the variation explained by the identified QTLs. The most significant QTL was found on chromosome V at 5.3 Mbp for the population growth on Sphingomonas, where the JU1931 allele increased population growth. For the body size traits, 1 to 2 QTLs were found explaining up to $16 \%$ of the phenotypic variation, with the exception of the length to width ratio for which 5 QTLs were found explaining 38\% of the variation. Overall, we found that QTLs were mostly determined by JU1511-specific (21 QTLs) and JU1941-specific (14 QTLs) SNPs and relatively few by other alleles. Comparison of major effect QTL locations for the different traits identifies no clear evidence of trade-offs between traits measured in this study and only limited evidence for genomic regions affecting multiple traits simultaneously (Fig. 7).

\section{Discussion}

We have developed a new C. elegans multi-parental recombinant inbred line population (mpRILs) derived from four wild-type strains capturing local genetic variation. This population of mpRILs complements existing RIL panels derived from two founders [29, 30, 43, 57,
59] and a multi-parent population derived from experimental evolution lines [26]. The four founding parental wild types originated from two different locations in France, Orsay (JU1511 and JU1941) and Santeuil (JU1926 and JU1931), collected and provided by Marie-Anne Félix and described in [25]. These strains were selected because of their genetic differences with each other. They also differed strongly compared to the two widely used strains Bristol N2 and Hawaii CB4856 [25]. Nevertheless, any small number of strains will only encompass a limited part of the total genetic variation in C. elegans. Yet, allele coverage will increase with an increasing number of RIL populations with different parental strains, thereby enhancing our overall ability to dissect the functional consequences and possible underlying evolutionary causes of global genetic variation.

Our mpRIL population is the first C. elegans population genotyped by SNPs in coding regions using RNA-seq. As RNA was isolated at a single time point (stage L4) during the life of the worm, these SNPs are limited to coding regions of genes that are expressed at that particular age. This limits the detection of SNPs to a temporally defined collection of expressed genes. There is a chance that this biases the detection of QTLs to SNPs of genes expressed at this point in the worm's 
life. However, comparing the SNP distribution to genomic SNPs in C. elegans, we show that the SNPs based on RNA-seq are distributed according to expectation $[10,29]$. Therefore, these SNPs are representative of the total genetic variation across the four strains. Hence, the QTLs detected are likely to be unbiased. Nonetheless, DNA sequencing would be beneficial to get better coverage in regions where coding SNPs are sparse and to detect the non-coding SNPs and other polymorphisms that can be potentially causal for observed phenotypic variation between the mpRILs.

Recombination frequencies detected based on RNA-seq are also not likely to be biased when compared to those from 2 parental RIL panels characterized by classical genetic markers or through genome sequencing. Yet, the average spacing between SNPs will be affected by RNA-seq compared to DNA-seq based. Our mpRIL population has a recombination frequency of 16.8 events per megabase pair and a mean introgression size of $5.0 \mathrm{Mbp}$. These 2 measures might be underestimated as the regions, where only non-coding variation exists between 2 or more lines recombination becomes impossible to detect. The previously described 2 parental RIAIL populations [29] showed a higher genome-wide recombination frequency of 36 per megabase pair, most likely due to the advanced inter-cross design that was used to generate the RIAILs [29]. Overall, we found approximately 9000 SNPs with an average spacing of $11 \mathrm{~kb}$ compared to 1454 nuclear SNP markers with a spacing of $61,160 \mathrm{bp}$ in the RIAIL population. A spacing of 1 SNP per $20 \mathrm{~kb}$ was reported for a multi-parental experimental evolution (CeMEE) panel, derived from 16 parental strains in C. elegans [26]. As far as these results can be compared, they show that all these populations are in the same range regarding SNP density, with an overall increase in SNP density for our mpRIL population.

Heritability values $(50-80 \%)$ are relatively high for some traits compared to those previously reported ( 20\%) [73] yet similar to heritability values found for related fitness traits across other species. Moreover, the values reported in our study are in line with those reported by Noble et al. [26]. It should be noted that heritability is a characteristic of a trait in a population and not of an individual trait in general. Therefore, heritability values are dependent on both the environmental and genetic circumstances of a population. Future studies with different RIL panels will help to understand to what extent heritability for specific traits can vary between populations of a species and whether and why there may be upper or lower boundaries for such measures. The range of inbred populations currently becoming available in $C$. elegans may be ideal to dissect variation in heritability in more detail.

We have investigated the genetic architecture of a range of complex traits and identified single-and usually
multiple-QTLs underlying the observed variation. Overall, QTLs with a JU1931 (4), JU1926 (4), or SDP 13 (2) allelic effect were found least frequently. QTLs with a JU1511 (21) and JU1941 (14) allelic effect were most abundant. This differential pattern of allelic effects highlights another advantage of using a mpRIL population over classical two parental RIL populations. Around 80\% of the QTLs would have been found when just JU1511 and JU1941 had been used as parental strains for a RIL population. In contrast, only 30\% of the QTLs would have been found if JU1926 and JU1931 served as parental strains. Furthermore, in the regions where multiple SDP overlap (left arm of chromosomes I, IV, and V; right arm of chromosomes I, IV, V, and X; and all of chromosome III), the number of potential causal SNPs can be reduced, as each QTL is mapped on a specific SDP. Based on the coding SNPs, the estimated reduction in potential causal SNPs was found to be $30-99 \%$ of the total SNPs on the QTL (Additional file 1: Table S8). It is tempting to speculate about local adaptation and niche formation as causal for the fact that most QTLs have a specific allelic effect from one of the Orsay strains (JU1511 or JU1941), yet it could be as simple as those lines having the most strain-specific (not shared) SNPs over the largest parts of the genome leading to more specific QTL effects.

We compared the detected QTLs in the mpRILs to those reported from other studies, revealing the identification of novel QTLs. For example, for variation in lifespan, in a two-parent N2 x CB4856 IL population, QTLs were detected on chromosomes II and IV [20] and in another study on chromosomes I, II, III, IV, and X [32] while in the current study, QTLs for the same trait were found on chromosomes I, III, IV, V, and X (Fig. 7)-without any apparent overlap. For lifespan under DR, we identified QTLs on chromosomes I, II, IV, V, and X, again at different positions than in previous publications where QTLs for lifespan under DR were found in lines derived from N2 and CB4856 [70]. Population growth on the different bacteria, including the toxic Bt strains, did not match with the QTLs found for leaving behavior on those the same bacteria [36]. QTLs for body size similarly differed between studies. Snoek et al. [20] detected a QTL for body size (length) on chromosome IV whereas we found QTLs in other locations. For body size (volume), we found a QTL on chromosome X whereas Gutteling et al. [73] reported QTLs on chromosomes IV and X. These examples emphasize that our new multi-parent RIL panel shows high power to identify previously undetected QTLs for complex traits.

Our findings support the idea that a substantial part of the variation in a range of complex traits in C. elegans is determined by QTLs of large effect rather than only by multiple QTLs of small effect. This is corroborated by other studies mapping complex traits in C. elegans. 
McGrath et al. [51] identified two QTLs associated with digenic behavior in response to environmental $\mathrm{O}_{2}$ and $\mathrm{CO}_{2}$ levels. Gaertner et al. [37] mapped a set of few loci determining thermal preference and isothermal dispersion and found these loci to interact epistatically, explaining $50 \%$ of the total variation. Andersen et al. [46] reported the detection of a few QTLs for lifetime fecundity and adult body size explaining up to $23 \%$ of the variation. In an extensive complex trait mapping study, Andersen et al. [41] investigated the loci underlying fecundity and multiple body size traits. For fecundity, they found a single QTL on chromosome IV explaining $12 \%$ of the phenotypic variance. Comparing these results with the results obtained from the mpRILs shows that multiple and different variations inducing alleles, even with a relatively small effect, are present within the total set of genetic variation in C. elegans. Moreover, the allelic effects might be dependent on the genetic background or epistatic interactions [26].

The nematode is likely subject to boom-and-bust demographics in nature, which may or may not coincide with natural selection. These demographics can enhance genetic differentiation over time and space. As a consequence, RILs generated from genotypes, which have not yet been considered in previous panels, are likely genetically distinct, possibly helping to enhance our general ability to map QTLs. Moreover, if boom-and-bust demographics coincide with strong natural selection, then this could at least temporally lead to local adaptation. This may be expected if populations from different habitat types are considered, as it is the case here, where habitats are either more natural (wood habitat) or shaped by human activities (orchard), most likely leading to differences in the associated microbial communities, possible parasites, predators, and competitors, and also abiotic parameters. If such differences in natural selection exist, then the resulting mpRIL panel may help to identify the genetic basis of local adaptation.

The advantage of mpRIL populations to the classical 2 parental RIL populations is that mpRILs capture a larger part of natural genetic variants in more combinations and hence cover these variants better. This is supported by similar multi-parent RIL studies in A. thaliana populations derived from 19 different parental accessions [64], and many more MAGIC populations were developed for different species, including mice [65]. In our mpRIL population, the polymorphisms show more patterns of segregations due to SNP distribution patterns between the parental strains, making candidate/causal gene selections more efficient. As each recombination event can break up 7 SNP distribution patterns, we found 57 informative breakpoints per megabase pair, which can drastically reduce the number of candidate causal polymorphisms. These numbers suggest that our new panel may help to characterize the genetic architecture of complex traits at a higher resolution.
Moreover, our developed 4-parent mpRIL population adds a new mapping tool for studying the complex trait architecture in the model species $C$. elegans and complements existing RIL panels using 2 parental strains and in case of the CeMEE panel 16 parents. Compared to the latter population, our mpRILs represent a relatively equal distribution of standing unperturbed local natural genetic variation as opposed to the genetic variation partially derived from laboratory selection experiments [26]. Taken together, our mpRIL population provides a straight forward and, in some cases, better performing alternative next to existing mapping panels.

\section{Conclusion}

Overall, multi-parent RIL populations have a higher number of informative SNP markers than the classic two parental RIL sets in a variety of organisms. We show that in our mpRIL population, the number of QTLs is likely to be increased as well as the distinction of candidate causal SNPs and therefore resolution for genetic characterization of complex traits.

\section{Methods}

\section{C. elegans strains, culturing, and crossing}

C. elegans strains were cultured at $20^{\circ} \mathrm{C}$ on OP50, unless specified otherwise for a specific screen or cross. For the construction of the multi-parental RIL, population lines were crossed as described in Fig. 1 and Additional file 1: Table S1 followed by 6 generations of single-worm descent inbreeding. Males were induced by heat stress (4-6 $\mathrm{h}$ at $\left.30{ }^{\circ} \mathrm{C}\right)$. To allow the 4 parental genomes (JU1511, JU1941, JU1926, and JU1931) [25] to recombine, we set up a crossing scheme in which 2 pairs of wild isolates were crossed and both the obtained $\mathrm{F} 1$ populations were reciprocally inter-crossed (Fig. 1; Additional file 1: Table S1). To enable crossovers on chromosome $\mathrm{X}$ and to generate extra crossovers, the heterozygous F2 obtained from these initial crosses were further inter-crossed. To create homozygous genotypes, single worms were selected from the F2 as well as from the F2 inter-cross for 6 generations of single-worm inbreeding. From these 383 lines, a population of 200 different multi-parental recombinant inbred lines (mpRILs) was randomly picked for mRNA sequencing to obtain the genetic variation in the coding sequence.

\section{RNA sequencing RNA isolation}

For each mpRIL and parental strain, worms were grown on two 6 -cm dishes at $16{ }^{\circ} \mathrm{C}$ on OP50 and bleached at the adult stage. The eggs were distributed over two $6-\mathrm{cm}$ dishes and grown at $24{ }^{\circ} \mathrm{C}$ for $48 \mathrm{~h}$ after which the animals were rinsed of the plates and flash frozen in liquid nitrogen. We isolated RNA from these samples using 
the Maxwell ${ }^{\circ} 16$ AS2000 instrument with a Maxwell $^{\circ} 16$ LEV simplyRNA Tissue Kit (both Promega Corporation, Madison, WI, USA). For isolation, the protocol was followed with a modified lysis step. In the lysis step, next to $200 \mu \mathrm{l}$ homogenization buffer and $200 \mu \mathrm{l}$ lysis buffer, $10 \mu \mathrm{l}$ of a $20-\mathrm{mg} / \mathrm{ml}$ stock solution of proteinase $\mathrm{K}$ was added to each sample. Subsequently, the samples were incubated for $10 \mathrm{~min}$ at $65^{\circ} \mathrm{C}$ and $1000 \mathrm{rpm}$ in a Thermomixer (Eppendorf, Hamburg, Germany). After cooling on ice for $1 \mathrm{~min}$, the standard protocol was followed.

\section{Sequencing}

We used standard Illumina protocols for the preparation and subsequent sequencing of RNA libraries. Libraries were sequenced on an Illumina HiSeq ${ }^{\mathrm{Ta}} 2000$ sequencing machine, using paired ends and 100 nucleotide read lengths. The raw data is available in the Sequence Read Archive (SRA; www.ncbi.nlm.nih.gov/bioproject/ PRJNA495983/) with ID PRJNA495983.

\section{SNP calling}

The untrimmed paired-end reads were mapped against the N2 reference genome (WS220) [74, 75] using Tophat [76], allowing for four read mismatches and a read edit distance of 4. SNPs were called using SAMtools [77] mpileup with bcftools and vcfutils, using a minimum of five mapped reads per SNP. Further selection and quality filters were used in the construction of the genetic map to minimize the number and effect of false SNPs.

\section{Construction of the genetic map}

To construct a genetic map from the SNPs detected in the RNA-seq data, we adjusted the method used in Serin et al. [78]. For this C. elegans population, we selected the SNPs by several parameters. First, we selected those SNPs present in at least one of the parental lines in both RNA sequence replicates (two populations from separate plates were sequenced per parental line). Further selection was made based on (i) the presence in the mpRILs $(\min =10$, $\max =180$, quality $>199$ ), (ii) correlation with neighboring SNPs of the same parental origin $(>0.8)$, and (iii) heterozygosity (<40 mpRILs). These SNPs (Additional file 1 : Table S2) were used directly in the SNP map of the population (Additional file 1: Table S3) or translated to the parental origin genetic map (Additional file 1: Table S4). For the parental origin map, stretches of ten SNPs were used to determine the parental origin and extended when inconclusive. In case of recombinations, breakpoints were put halfway between the determining SNPs.

\section{Phenotyping}

\section{Population growth}

Orsay/Santeuil mpRIL population growth was measured as the total offspring of three L4 hermaphrodites after
5 days at $20{ }^{\circ} \mathrm{C}$ in liquid peptone free medium (PFM). 24-well plates were inoculated with $1 \mathrm{ml}$ liquid PFM per well and food bacteria added to a final $\mathrm{OD}_{600}$ of 5 . The six different bacterial treatments were (i) Escherichia coli OP50, (ii) Erwinia rhapontici (isolated from Orsay, France), (iii) Sphingobacterium sp. (isolated from Orsay, France), (iv) a non-pathogenic Bacillus thuringiensis strain DSM-350E, and a pathogenic Bacillus thuringiensis strain NRRL B-18247 in the two concentrations of (v) 1:300 and (vi) 1:600. After 5 days, worms were fixed in $4 \%$ formaldehyde and stored at $8{ }^{\circ} \mathrm{C}$ until counting [25].

\section{Lifespan assays}

Worm lifespan assays were performed at $20{ }^{\circ} \mathrm{C}$, with populations initiated from synchronized larvae isolated by incubating the eggs from sodium hypochlorite-treated gravid adults on plates without a food source [42]. After $24 \mathrm{~h}$, the plates were seeded with Escherichia coli OP50 as a food source and the worms were allowed to grow, en masse, for $48 \mathrm{~h}$ to the L4/young adult's stage. After $48 \mathrm{~h}$, ad libitum (normal lifespan) worms were moved to fresh seeded standard NGM plates (5 worms per plate and 8 plates per treatment). The lifespan under DR worms was moved to seeded PFM plates (5 worms per plate and 8 plates per treatment). The method of total withdrawal of peptone from the agarose plates is a relatively mild form of DR, as described by Stastna et al. [70]. To test the lifespan, worms were observed daily, with nematodes transferred to new plates every day until reproduction had ceased as assays were performed without the use of FUdR. After the reproductive period, the DR worms were moved to fresh plates every other day to prevent food deprivation. Worms were considered to have died if they were not moving and failed to respond to touch with a worm pick. Any worms that died due to maternal hatching (bagging) were censored out of the analysis of lifespan. Each mpRIL within an experimental block was tested at the same time under both conditions, with a total of 40 worms per treatment per mpRIL; plates were then randomized and blind coded. The movement of the L4/young adult worms to fresh plates was counted as day 1 for all the lifespan measurements. In total, the mpRILs were assayed in 6 blocks with 35-48 formally randomly selected mpRILs in each block, with some mpRILs present in multiple blocks. RILs were not included in the analysis if the lifespan of less than 3 worms was observed per treatment. In addition to the mpRILs, the 4 parental lines and N2 were also tested in all lifespan assays.

\section{Heat shock resistance}

Worms were cultured at $15{ }^{\circ} \mathrm{C}$ prior to the heat shock assays. Worms were synchronized as for the lifespan assay and allowed to grow en masse to L4/young adult stage $[42,79,80]$. At this stage, worms were transferred 
to fresh plates, ten worms per plate with five replicates for each of the mpRILs and each of the parental strains. The plates were then randomized and blind coded. Worms were then placed at $35^{\circ} \mathrm{C}$ for $10 \mathrm{~h}$. After the heat shock, worms were allowed to rest at $15^{\circ} \mathrm{C}$ for $48 \mathrm{~h}$ before scoring for survival, when worms that did not respond to a gentle prod with the worm pick were scored as dead. Worms that crawled off the plates or died of bagging were censored from the experiment. The data were then converted into a proportion of survival.

\section{Oxidative stress resistance}

Worms were maintained at $20^{\circ} \mathrm{C}$, synchronized as described above, and grown en masse to the L4/young adult's stage. After $48 \mathrm{~h}$, the worms were washed off the plates with M9 buffer and 10-30 individuals were transferred to 96-well plates in a total volume of $48 \mu \mathrm{l}$, with 3 replicates for all the mpRILs and N2. The plates were then transferred to a WMicrotracker-One $^{\mathrm{Tm}}$ (PhylumTech), and activity over $30 \mathrm{~min}$ was determined at $20^{\circ} \mathrm{C}$. After this step, $2 \mu \mathrm{l}$ of $0.4 \%$ $\mathrm{H}_{2} \mathrm{O}_{2}$ solution was added to all wells, giving a final volume of $50 \mu \mathrm{l}$, except for the control, which had $2 \mu \mathrm{l}$ of M9 buffer added to make up the final volume. Worms were then incubated for $24 \mathrm{~h}$ at $20^{\circ} \mathrm{C}$. After $24 \mathrm{~h}$, the locomotive activity of the worms was measured again. WMicrotracker-One ${ }^{\mathrm{mm}}$ records the movement as photo beam interruptions within wells of 96-well plates. The data were then processed as follows: (activity of the wells before - activity after $24 \mathrm{~h}$ )/before $=$ activity score. An activity score of -1 represents no movement and hence that all worms were dead at the end of the treatment, and an activity score of 0 indicates an activity level after $24 \mathrm{~h}$ that is the same as before. This score can also generate values above 0 , which indicates that worms were more active after the hydrogen peroxide treatment.

\section{Developmental time and occurrence of males}

Starvation-synchronized L1 juveniles of the mpRILs and parental strains were grown on E. coli OP50 at $24{ }^{\circ} \mathrm{C}$ and after $48 \mathrm{~h}$ inspected at 1 - $\mathrm{h}$ time intervals. Developmental time was defined as the period between synchronized hatching and time until the first eggs. Time until first egg scoring was adjusted from $[25,81]$ by placing $20-40$ worms on NGM, done in duplo, and scoring every hour starting at $48 \mathrm{~h}$ until $54 \mathrm{~h}$ for eggs. This was done on $E$. coli OP50 and Erwinia rhapontici bacteria previously used to measure the time until the first egg in multiple wild isolates [25]. We scored the time of the first egg visible on plate and the time when multiple groups of $\sim 10$ eggs were visible. Averages of these time points per mpRIL were used in QTL mapping. Moreover, the occurrence of males on the plates was recorded after population growth and used for QTL mapping.

\section{Size and volume}

Analysis of the length and width of young gravid adults of the mpRILs was performed with a particle analyzer (RapidVue; Beckman Coulter Inc., Miami, FL, USA) [25]. Phenotypic size and volume data for the parental strains could not be recorded together with the mpRILs. The size and volume of the parental strains were measured in Volkers et al. 2013, yet due to the between-batch variation, these measurements are incomparable to the mpRILs measured in this study. The parental measurements were not needed for the QTL mapping.

\section{QTL mapping}

We started with single-marker mapping for each trait to find the SNP with the most significant QTL (Additional file 5: Figure S4). This SNP was used as the starting point in the forward mapping approach. Forward mapping was done by selecting co-factors one by one, starting with the most significant and remap with that co-factor, and selecting the next most significant SNP until no more SNP was present with a $p<0.001$ or a maximum of ten co-factors was reached. Then, QTLs were remapped with the selected co-factors and an exclusion window of $2 \mathrm{Mbp}$. Co-factors within this window were excluded from the mapping model when QTLs were mapped in the window (Additional file 4: Figure S3). Obtained QTLs were determined significant when $-\log 10(p)>3$ and borders were determined at the point where the QTL profile drops $2-\log 10(p)$ scores below the peak. (Permutations showed maximum QTLs ranging from $-\log 10(p)$ of 2.1 to 3.9 , with the exception of the traits describing the occurrence of males on the plate for which in a number of permutations, $a-\log 10(p)$ was found $>4.5)$. All QTL profiles can be obtained and interactively explored in EleQTL (www.bioinformatics.nl/EleQTL). The heritability for each trait was calculated by dividing the variation between the mpRILs by the total variation.

\section{Additional files}

\begin{abstract}
Additional file 1: Table S1. Detailed crossing scheme used to make the mpRILs. The crosses from which each individual mpRIL was made can be found here. Table S2. SNP info. SNP position and SNP distribution pattern (SDP). Table S3. SNP genetic map. SNP identity per mpRIL. Table S4. Parental background genetic map. Genome-wide parental background for each individual mpRIL. Table S5. Average phenotypes per mpRIL used for QTL mapping. Including WN and sequence identifiers. Table S6. Trait descriptive. Number of mpRILs for which the phenotype was measured, minimum trait value, maximum trait value, mean trait value, median trait value, trait value of parental line JU1511, trait value of parental line JU1926, trait value of parental line JU1931, trait value of parental line JU1941, heritability, heritability type, number of QTLs found, and explained variation by QTLs. Table S7. Correlation between traits. Pearson correlation of the mean traits values in the mpRIL population. Table S8. Identified QTLs. (XLSX $11929 \mathrm{~kb})$
\end{abstract}

Additional file 2: Figure S1. Distribution of parental alleles in the multiparental recombinant inbred lines. Colors indicate the percentage of parental occurrence per genomic position ( $x$-axis) per cross ( $y$-axis) as estimated from 
the parental SNP distribution patterns (SDP). Chromosomes are in separate panels on the $x$-axis. mpRILs are grouped according to their cross history. Group Z is the parental lines. (PDF $536 \mathrm{~kb}$ )

Additional file 3: Figure S2. SNP distribution pattern (SDP) per mpRIL. For each of the seven SDPs, the genotype for each mpRIL is shown. SDPs are shown on top, chromosomes on the right. The genotype of the mpRIL is green when it has the SNP corresponding to the SDP and red when it has the opposite variant. SDP 12 (JU1511/JU1926 vs JU1931/ JU1941), 13 (JU1511/JU1931 vs JU1926/JU1941), 14 (JU1511/JU1941 vs JU1926/JU1931), JU1511 (JU1511 vs rest), JU1926 (JU1926 vs rest), JU1931 (JU1931 vs rest), and JU1941 (JU1941 vs rest). Notice that each recombination event can break up multiple SDP. (TIFF $11718 \mathrm{~kb}$ )

Additional file 4: Figure S3. Forward mapping QTL profiles for each trait. Trait names are shown on the right. Chromosome number is shown on top. Genomic position in megabase pair is shown on the $x$-axis. For each SNP, the significance in $-\log 10(p)$ is multiplied by the sign of the effect on the $y$-axis. Colors indicate SPD of the SNP and triangle the co-factors used in the final model of the forward mapping approach. (PDF $883 \mathrm{~kb}$ )

Additional file 5: Figure S4. Single marker QTL profiles for each trait. Trait names are shown as title. Chromosome number is shown on top. Genomic position in megabase pair is shown on the $x$-axis. For each SNP, the significance in $-\log 10(p)$ is multiplied by the sign of the effect on the $y$-axis. Colors indicate SPD of the SNP. (PDF $891 \mathrm{~kb}$ )

\section{Acknowledgements}

We thank Marie-Anne Félix for providing the parental lines used to make the mpRILs. We thank the compilers of WormBase for making it a versatile and important resource for C. elegans.

\section{Funding}

We acknowledge financial support from the Deutsche Forschungsgemeinschaft to HS, grant number SCHU 1415/11 and project Al within the CRC 1182. Furthermore, financial support from the NWO-ALW (project 855.01.151) to RJMV.

\section{Availability of data and materials}

Phenotypic data generated and analysed during this study are included in this published article and its supplementary information files. The mpRIL strains are available upon request from J.E. Kammenga and H. Schulenburg. Sequencing data is available from the NCBI Sequence Read Archive database (SRA; www.ncbi.nlm.nih.gov/bioproject/PRJNA495983/), project ID PRJNA495983. QTL profiles and phenotypic data can be found and explored interactively at EleQTLNormQTL2.0 (www.bioinformatics.nl/EleQTL).

\section{Authors' contributions}

LBS, HS, SCH, and JEK conceived the study. LBS, HN, and MGS analyzed the data. RJMV, BPB, CP, PD, RN, JR, PR, and JJS performed the experiments and/or provided the phenotypic data. LBS and JEK wrote the paper with contributions from all authors. All authors read and approved the final manuscript.

\section{Ethics approval and consent to participate}

Not applicable

\section{Competing interests}

The authors declare that they have no competing interests. The funders had no role in study design, data collection and analysis, decision to publish, or preparation of the manuscript.

\section{Publisher's Note}

Springer Nature remains neutral with regard to jurisdictional claims in published maps and institutional affiliations.

\section{Author details}

'Laboratory of Nematology, Wageningen University, Droevendaalsesteeg 1, NL-6708 PB Wageningen, The Netherlands. ${ }^{2}$ Theoretical Biology and Bioinformatics, Utrecht University, Padualaan 8, 3584 CH Utrecht, The Netherlands. ${ }^{3}$ Bioinformatics Group, Wageningen University, Droevendaalsesteeg 1, NL-6708 PB Wageningen, The Netherlands. ${ }^{4}$ Biomolecular Research Group, School of Human and Life Sciences, Canterbury Christ Church University, North Holmes Road, Canterbury CT1
1QU, UK. ${ }^{5}$ Department of Biology, Ghent University, K. L. Ledeganckstraat 35, B-9000 Ghent, Belgium. ' $Z$ Zoological Institute, University of Kiel, 24098 Kiel, Germany. ${ }^{7}$ Institute for Clinical Molecular Biology, University of Kiel, 24098 Kiel, Germany. ${ }^{8}$ Max Planck Institute for Evolutionary Biology,

August-Thienemann-Str. 2, 24306 Plön, Germany.

Received: 17 October 2018 Accepted: 26 February 2019

Published online: 12 March 2019

\section{References}

1. Petersen C, Dirksen P, Schulenburg H. Why we need more ecology for genetic models such as C. elegans. Trends Genet. 2015;31(3):120-7.

2. Viney M, Harvey S. Reimagining pheromone signalling in the model nematode Caenorhabditis elegans. PLoS Genet. 2017;13(11):e1007046.

3. Lynch M, Conery JS. The origins of genome complexity. Science. 2003; 302(5649):1401-4.

4. Saltz JB, Bell AM, Flint J, Gomulkiewicz R, Hughes KA, Keagy J. Why does the magnitude of genotype-by-environment interaction vary? Ecol Evol. 2018; 8(12):6342-53

5. Mackay TF, Stone EA, Ayroles JF. The genetics of quantitative traits: challenges and prospects. Nat Rev Genet. 2009;10(8):565-77.

6. Lynch M, Walsh B: The origins of genome architecture. Sunderland: Sinauer Associates, Inc:; 2007.

7. Rockman MV. Reverse engineering the genotype-phenotype map with natural genetic variation. Nature. 2008;456(7223):738-44.

8. Gaertner BE, Phillips PC. Caenorhabditis elegans as a platform for molecular quantitative genetics and the systems biology of natural variation. Genet Res. 2010;92(5-6):331-48.

9. Sterken MG, Snoek LB, Kammenga JE, Andersen EC. The laboratory domestication of Caenorhabditis elegans. Trends Genet. 2015;31(5):224-31.

10. Andersen EC, Gerke JP, Shapiro JA, Crissman JR, Ghosh R, Bloom JS, Felix MA, Kruglyak L. Chromosome-scale selective sweeps shape Caenorhabditis elegans genomic diversity. Nat Genet. 2012:44(3):285-90.

11. Frezal L, Felix MA. C. elegans outside the petri dish. eLife. 2015;4:e05849. https://doi.org/10.7554/eLife.05849.

12. Schulenburg H, Felix MA. The natural biotic environment of Caenorhabditis elegans. Genetics. 2017;206(1):55-86.

13. Lee D, Yang H, Kim J, Brady S, Zdraljevic S, Zamanian M, Kim H, Paik YK, Kruglyak L, Andersen EC, et al. The genetic basis of natural variation in a phoretic behavior. Nat Commun. 2017:8(1):273.

14. Lee H, Choi MK, Lee D, Kim HS, Hwang H, Kim H, Park S, Paik YK, Lee J. Nictation, a dispersal behavior of the nematode Caenorhabditis elegans, is regulated by IL2 neurons. Nature Neurosci. 2011;15(1):107-12.

15. Barriere A, Felix MA. Temporal dynamics and linkage disequilibrium in natural Caenorhabditis elegans populations. Genetics. 2007:176(2):999-1011.

16. Petersen C, Saebelfeld M, Barbosa C, Pees B, Hermann RJ, Schalkowski R, Strathmann EA, Dirksen $P$, Schulenburg $H$. Ten years of life in compost: temporal and spatial variation of North German Caenorhabditis elegans populations. Ecol Evol. 2015;5(16):3250-63.

17. Barriere A, Felix MA. High local genetic diversity and low outcrossing rate in Caenorhabditis elegans natural populations. Curr Biol. 2005;15(13):1176-84

18. Richaud A, Zhang G, Lee D, Lee J, Felix MA. The local coexistence pattern of selfing genotypes in Caenorhabditis elegans natural metapopulations. Genetics. 2018:208(2):807-21.

19. Seidel HS, Rockman MV, Kruglyak L. Widespread genetic incompatibility in C. elegans maintained by balancing selection. Science. 2008:319(5863):589-94.

20. Snoek LB, Orbidans HE, Stastna JJ, Aartse A, Rodriguez M, Riksen JA, Kammenga JE, Harvey SC. Widespread genomic incompatibilities in Caenorhabditis elegans. G3. 2014;4(10):1813-23.

21. Dolgin ES, Charlesworth B, Baird SE, Cutter AD. Inbreeding and outbreeding depression in Caenorhabditis nematodes. Evolution. 2007:61(6):1339-52.

22. Ashe A, Belicard T, Le Pen J, Sarkies P, Frezal L, Lehrbach NJ, Felix MA, Miska EA. A deletion polymorphism in the Caenorhabditis elegans RIG-I homolog disables viral RNA dicing and antiviral immunity. eLife. 2013;2:e00994.

23. Greene JS, Dobosiewicz M, Butcher RA, McGrath PT, Bargmann Cl. Regulatory changes in two chemoreceptor genes contribute to a Caenorhabditis elegans QTL for foraging behavior. eLife. 2016;5:e21454. https://doi.org/10.7554/eLife.21454.

24. Haber M, Schungel M, Putz A, Muller S, Hasert B, Schulenburg $\mathrm{H}$, Evolutionary history of Caenorhabditis elegans inferred from microsatellites: 
evidence for spatial and temporal genetic differentiation and the occurrence of outbreeding. Mol Biol Evol. 2005;22(1):160-73.

25. Volkers RJ, Snoek LB, Hubar CJ, Coopman R, Chen W, Yang W, Sterken MG, Schulenburg H, Braeckman BP, Kammenga JE. Gene-environment and protein-degradation signatures characterize genomic and phenotypic diversity in wild Caenorhabditis elegans populations. BMC Biol. 2013;11:93.

26. Noble LM, Chelo I, Guzella T, Afonso B, Riccardi DD, Ammerman P, Dayarian A, Carvalho S, Crist A, Pino-Querido A, et al. Polygenicity and epistasis underlie fitness-proximal traits in the Caenorhabditis elegans multiparental experimental evolution (CeMEE) panel. Genetics. 2017;207(4):1663-85.

27. Teotonio H, Estes S, Phillips PC, Baer CF. Experimental evolution with Caenorhabditis nematodes. Genetics. 2017;206(2):691-716.

28. Seidel HS, Ailion M, Li J, van Oudenaarden A, Rockman MV, Kruglyak L. A novel sperm-delivered toxin causes late-stage embryo lethality and transmission ratio distortion in C. elegans. PLoS Biol. 2011;9(7):e1001115.

29. Rockman MV, Kruglyak L. Recombinational landscape and population genomics of Caenorhabditis elegans. PLoS Genet. 2009;5(3):e1000419.

30. Li Y, Alvarez OA, Gutteling EW, Tijsterman M, Fu J, Riksen JA, Hazendonk E, Prins P, Plasterk RH, Jansen RC, et al. Mapping determinants of gene expression plasticity by genetical genomics in C. elegans. PLoS Genet. 2006; 2(12):e222.

31. Thompson OA, Snoek LB, Nijveen H, Sterken MG, Volkers RJ, Brenchley R, Van't Hof A, Bevers RP, Cossins AR, Yanai I, et al. Remarkably divergent regions punctuate the genome assembly of the Caenorhabditis elegans Hawailian strain CB4856. Genetics. 2015;200(3):975-89.

32. Doroszuk A, Snoek LB, Fradin E, Riksen J, Kammenga J. A genome-wide library of CB4856/N2 introgression lines of Caenorhabditis elegans. Nucleic Acids Res. 2009;37(16):e110.

33. Li Y, Breitling R, Snoek LB, van der Velde KJ, Swertz MA, Riksen J, Jansen RC, Kammenga JE. Global genetic robustness of the alternative splicing machinery in Caenorhabditis elegans. Genetics. 2010;186(1):405-10.

34. Cook DE, Zdraljevic S, Tanny RE, Seo B, Riccardi DD, Noble LM, Rockman MV, Alkema MJ, Braendle C, Kammenga JE, et al. The genetic basis of natural variation in Caenorhabditis elegans telomere length. Genetics. 2016; 204(1):371-83.

35. Gao AW, Sterken MG, Uit de Bos J, van Creij J, Kamble R, Snoek BL, Kammenga JE, Houtkooper RH. Natural genetic variation in C. elegans identified genomic loci controlling metabolite levels. Genome Res. 2018; 28(9):1296-308,

36. Nakad R, Snoek LB, Yang W, Ellendt S, Schneider F, Mohr TG, Rosingh L, Masche AC, Rosenstiel PC, Dierking K, et al. Contrasting invertebrate immune defense behaviors caused by a single gene, the Caenorhabditis elegans neuropeptide receptor gene npr-1. BMC Genomics. 2016;17:280.

37. Gaertner BE, Parmenter MD, Rockman MV, Kruglyak L, Phillips PC. More than the sum of its parts: a complex epistatic network underlies natural variation in thermal preference behavior in Caenorhabditis elegans. Genetics. 2012; 192(4):1533-42.

38. Zhu Z, Lu Q, Zeng F, Wang J, Huang S. Compatibility between mitochondrial and nuclear genomes correlates with the quantitative trait of lifespan in Caenorhabditis elegans. Sci Rep. 2015;5:17303.

39. Singh KD, Roschitzki B, Snoek LB, Grossmann J, Zheng X, Elvin M, Kamkina P, Schrimpf SP, Poulin GB, Kammenga JE, et al. Natural genetic variation influences protein abundances in C. elegans developmental signalling pathways. PloS one. 2016;11(3):e0149418.

40. Evans KS, Brady SC, Bloom JS, Tanny RE, Cook DE, Giuliani SE, Hippleheuser SW, Zamanian M, Andersen EC. Shared genomic regions underlie natural variation in diverse toxin responses. Genetics. 2018; 210(4):1509-25

41. Andersen EC, Shimko TC, Crissman JR, Ghosh R, Bloom JS, Seidel HS, Gerke $J P$, Kruglyak L. A powerful new quantitative genetics platform, combining Caenorhabditis elegans high-throughput fitness assays with a large collection of recombinant strains. G3. 2015;5(5):911-20.

42. Rodriguez M, Snoek LB, Riksen JA, Bevers RP, Kammenga JE. Genetic variation for stress-response hormesis in C. elegans lifespan. Exp Gerontol. 2012;47(8):581-7.

43. Harvey SC, Shorto A, Viney ME. Quantitative genetic analysis of lifehistory traits of Caenorhabditis elegans in stressful environments. BMC Evol Biol. 2008;8:15.

44. Green JW, Snoek LB, Kammenga JE, Harvey SC. Genetic mapping of variation in dauer larvae development in growing populations of Caenorhabditis elegans. Heredity. 2013;111(4):306-13.
45. Vinuela A, Snoek LB, Riksen JA, Kammenga JE. Genome-wide gene expression regulation as a function of genotype and age in C. elegans. Genome Res. 2010;20(7):929-37.

46. Andersen EC, Bloom JS, Gerke JP, Kruglyak L. A variant in the neuropeptide receptor npr-1 is a major determinant of Caenorhabditis elegans growth and physiology. PLoS Genet. 2014;10(2):e1004156.

47. Ghosh R, Andersen EC, Shapiro JA, Gerke JP, Kruglyak L. Natural variation in a chloride channel subunit confers avermectin resistance in C. elegans. Science. 2012;335(6068):574-8.

48. Large EE, Xu W, Zhao Y, Brady SC, Long L, Butcher RA, Andersen EC, McGrath PT. Selection on a subunit of the NURF chromatin remodeler modifies life history traits in a domesticated strain of Caenorhabditis elegans. PLoS Genet. 2016;12(7):e1006219.

49. Reddy KC, Andersen EC, Kruglyak L, Kim DH. A polymorphism in npr-1 is a behavioral determinant of pathogen susceptibility in C. elegans. Science. 2009;323(5912):382-4.

50. Kammenga JE, Doroszuk A, Riksen JA, Hazendonk E, Spiridon L, Petrescu AJ, Tijsterman M, Plasterk RH, Bakker J. A Caenorhabditis elegans wild type defies the temperature-size rule owing to a single nucleotide polymorphism in tra-3. PLoS Genet. 2007;3(3):e34.

51. McGrath PT, Rockman MV, Zimmer M, Jang H, Macosko EZ, Kruglyak L, Bargmann Cl. Quantitative mapping of a digenic behavioral trait implicates globin variation in C. elegans sensory behaviors. Neuron. 2009;61(5):692-9.

52. Bendesky A, Tsunozaki M, Rockman MV, Kruglyak L, Bargmann Cl. Catecholamine receptor polymorphisms affect decision-making in C. elegans. Nature. 2011;472(7343):313-8.

53. Sterken MG, van Bemmelen van der Plaat $L$, JAG R, Rodriguez M, Schmid T, Hajnal A, Kammenga JE, Snoek BL. Ras/MAPK modifier loci revealed by eQTL in Caenorhabditis elegans. G3. 2017;7(9):3185-93.

54. Elvin M, Snoek LB, Frejno M, Klemstein U, Kammenga JE, Poulin GB. A fitness assay for comparing RNAi effects across multiple C. elegans genotypes. BMC Genomics. 2011;12:510.

55. Bendesky A, Pitts J, Rockman MV, Chen WC, Tan MW, Kruglyak L, Bargmann $\mathrm{Cl}$. Long-range regulatory polymorphisms affecting a GABA receptor constitute a quantitative trait locus (QTL) for social behavior in Caenorhabditis elegans. PLoS Genet. 2012;8(12):e1003157.

56. Noble LM, Chang AS, McNelis D, Kramer M, Yen M, Nicodemus JP, Riccardi DD, Ammerman P, Phillips M, Islam T, et al. Natural variation in plep-1 causes male-male copulatory behavior in C. elegans. Curr Biol. 2015;25(20):2730-7.

57. Shmookler Reis RJ, Kang P, Ayyadevara S. Quantitative trait loci define genes and pathways underlying genetic variation in longevity. Exp Gerontol. 2006; 41(10):1046-54

58. McGrath PT, Xu Y, Ailion M, Garrison JL, Butcher RA, Bargmann Cl. Parallel evolution of domesticated Caenorhabditis species targets pheromone receptor genes. Nature. 2011:477(7364):321-5.

59. Duveau F, Felix MA. Role of pleiotropy in the evolution of a cryptic developmental variation in Caenorhabditis elegans. PLoS Biol. 2012; 10(1):e1001230.

60. Schmid T, Snoek LB, Frohli E, van der Bent ML, Kammenga J, Hajnal A. Systemic regulation of RAS/MAPK signaling by the serotonin metabolite 5HIAA. PLoS Genet. 2015;11(5):e1005236.

61. Frezal L, Demoinet E, Braendle C, Miska E, Felix MA. Natural genetic variation in a multigenerational phenotype in C. elegans. Curr Biol. 2018;28(16):2588-96.

62. Teotonio H, Carvalho S, Manoel D, Roque M, Chelo IM. Evolution of outcrossing in experimental populations of Caenorhabditis elegans. PLoS One. 2012; (4):e35811.

63. King EG, Merkes CM, McNeil CL, Hoofer SR, Sen S, Broman KW, Long AD, Macdonald SJ. Genetic dissection of a model complex trait using the Drosophila synthetic population resource. Genome Res. 2012;22(8):1558-66.

64. Kover PX, Valdar W, Trakalo J, Scarcelli N, Ehrenreich IM, Purugganan MD, Durrant C, Mott R, Multiparent Advanced A. Generation inter-cross to fine-map quantitative traits in Arabidopsis thaliana. PLoS Genet. 2009;5(7):e1000551.

65. de Koning DJ, McIntyre LM. Back to the future: multiparent populations provide the key to unlocking the genetic basis of complex traits. Genetics. 2017;206(2):527-9.

66. Samuel BS, Rowedder H, Braendle C, Felix MA, Ruvkun G. Caenorhabditis elegans responses to bacteria from its natural habitats. Proc Natl Acad Sci U S A. 2016;113(27):E3941-9.

67. Dirksen P, Marsh SA, Braker I, Heitland N, Wagner S, Nakad R, Mader S, Petersen $C$, Kowallik $V$, Rosenstiel $P$, et al. The native microbiome of the nematode 
Caenorhabditis elegans: gateway to a new host-microbiome model. BMC Biol. 2016;14:38.

68. Rockman MV, Skrovanek SS, Kruglyak L. Selection at linked sites shapes heritable phenotypic variation in C. elegans. Science. 2010;330(6002):372-6.

69. Cutter AD, Payseur BA. Selection at linked sites in the partial selfer Caenorhabditis elegans. Mol Biol Evol. 2003;20(5):665-73.

70. Stastna JJ, Snoek LB, Kammenga JE, Harvey SC. Genotype-dependent lifespan effects in peptone deprived Caenorhabditis elegans. Sci Rep. 2015; 5:16259.

71. Liao CY, Rikke BA, Johnson TE, Diaz V, Nelson JF. Genetic variation in the murine lifespan response to dietary restriction: from life extension to life shortening. Aging Cell. 2010;9(1):92-5.

72. Snoek LB, Sterken MG, Volkers RJ, Klatter M, Bosman KJ, Bevers RP, Riksen JA, Smant G, Cossins AR, Kammenga JE. A rapid and massive gene expression shift marking adolescent transition in C. elegans. Scientific Reports. 2014;4:3912.

73. Gutteling EW, Doroszuk A, Riksen JA, Prokop Z, Reszka J, Kammenga JE. Environmental influence on the genetic correlations between life-history traits in Caenorhabditis elegans. Heredity. 2007;98(4):206-13.

74. Williams CR, Baccarella A, Parrish JZ, Kim CC. Trimming of sequence reads alters RNA-Seq gene expression estimates. BMC Bioinformatics. 2016;17:103.

75. Del Fabbro C, Scalabrin S, Morgante M, Giorgi FM. An extensive evaluation of read trimming effects on Illumina NGS data analysis. PLoS One. 2013;8(12):e85024.

76. Trapnell C, Pachter L, Salzberg SL. TopHat: discovering splice junctions with RNA-Seq. Bioinformatics. 2009:25(9):1105-11.

77. Li H, Handsaker B, Wysoker A, Fennell T, Ruan J, Homer N, Marth G, Abecasis G, Durbin R, Genome Project Data Processing S: The sequence alignment/ map format and SAMtools. Bioinformatics 2009, 25(16):2078-2079.

78. Serin EAR, Snoek LB, Nijveen H, Willems LAJ, Jimenez-Gomez JM, Hilhorst HWM, Ligterink W. Construction of a high-density genetic map from RNASeq data for an Arabidopsis bay-0 x Shahdara RIL population. Front Genet. 2017:8:201.

79. Snoek BL, Sterken MG, Bevers RPJ, Volkers RJM, Van't Hof A, Brenchley R, Riksen JAG, Cossins A, Kammenga JE. Contribution of trans regulatory eQTL to cryptic genetic variation in C. elegans. BMC Genomics. 2017;18(1):500.

80. Jovic K, Sterken MG, Grilli J, Bevers RPJ, Rodriguez M, Riksen JAG, Allesina S, Kammenga JE, Snoek LB. Temporal dynamics of gene expression in heatstressed Caenorhabditis elegans. PLoS One. 2017;12(12):e0189445.

81. van der Bent ML, Sterken MG, Volkers RJ, Riksen JA, Schmid T, Hajnal A, Kammenga JE, Snoek LB. Loss-of-function of beta-catenin bar-1 slows development and activates the Wnt pathway in Caenorhabditis elegans. Sci Rep. 2014;4:4926.

Ready to submit your research? Choose BMC and benefit from:

- fast, convenient online submission

- thorough peer review by experienced researchers in your field

- rapid publication on acceptance

- support for research data, including large and complex data types

- gold Open Access which fosters wider collaboration and increased citations

- maximum visibility for your research: over $100 \mathrm{M}$ website views per year

At $\mathrm{BMC}$, research is always in progress.

Learn more biomedcentral.com/submissions 